\title{
Initial Evaluation of a New Electromechanical Cooler for Safeguards Applications
}

September 2002

R. L. Coleman, J. S. Bogard and M. E. Murray 


\title{
DOCUMENT AVAILABILITY
}

Reports produced after January 1, 1996, are generally available free via the U.S. Department of Energy (DOE) Information Bridge:

Web site: http://www.osti.gov/bridge

Reports produced before January 1, 1996, may be purchased by members of the public from the following source:

\author{
National Technical Information Service \\ 5285 Port Royal Road \\ Springfield, VA 22161 \\ Telephone: 703-605-6000 (1-800-553-6847) \\ TDD: $703-487-4639$ \\ Fax: 703-605-6900 \\ E-mail: info@ntis.fedworld.gov \\ Web site: http://www.ntis.gov/support/ordernowabout.htm
}

Reports are available to DOE employees, DOE contractors, Energy Technology Data Exchange (ETDE) representatives, and International Nuclear Information System (INIS) representatives from the following source:

Office of Scientific and Technical Information

P.O. Box 62

Oak Ridge, TN 37831

Telephone: 865-576-8401

Fax: 865-576-5728

E-mail: reports@adonis.osti.gov

Web site: http://www.osti.gov/contact.html

This report was prepared as an account of work sponsored by an agency of the United States Government. Neither the United States government nor any agency thereof, nor any of their employees, makes any warranty, express or implied, or assumes any legal liability or responsibility for the accuracy, completeness, or usefulness of any information, apparatus, product, or process disclosed, or represents that its use would not infringe privately owned rights. Reference herein to any specific commercial product, process, or service by trade name, trademark, manufacturer, or otherwise, does not necessarily constitute or imply its endorsement, recommendation, or favoring by the United States Government or any agency thereof. The views and opinions of authors expressed herein do not necessarily state or reflect those of the United States Government or any agency thereof. 


\title{
INITIAL EVALUATION OF A NEW ELECTROMECHANICAL COOLER FOR SAFEGUARDS
} APPLICATIONS

\author{
R. L. Coleman, J. S. Bogard and M. E. Murray
}

September 2002

Prepared by

OAK RIDGE NATIONAL LABORATORY

P.O. Box 2008

Oak Ridge, Tennessee 37831-6285

managed by

UT-Battelle, LLC

for the

U.S. DEPARTMENT OF ENERGY

under contract DE-AC05-00OR22725 



\section{CONTENTS}

Page

LIST OF FIGURES $\ldots \ldots \ldots \ldots \ldots \ldots \ldots \ldots \ldots \ldots \ldots \ldots \ldots \ldots \ldots \ldots \ldots \ldots \ldots \ldots \ldots \ldots$

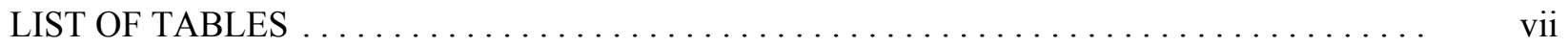

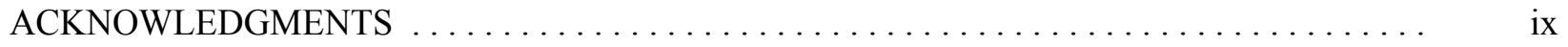

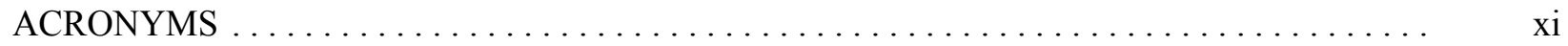

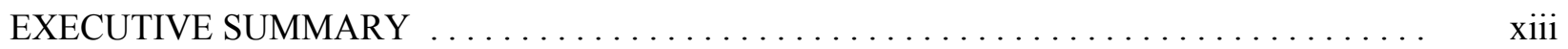

1. INTRODUCTION $\ldots \ldots \ldots \ldots \ldots \ldots \ldots \ldots \ldots \ldots \ldots \ldots \ldots \ldots \ldots \ldots \ldots \ldots \ldots \ldots \ldots \ldots \ldots$

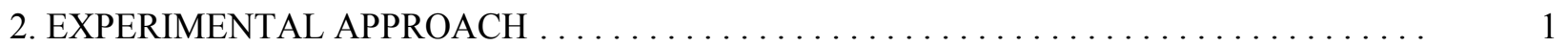

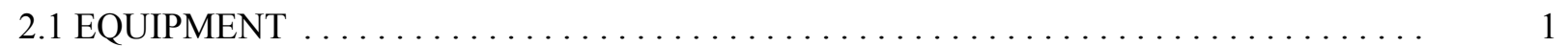

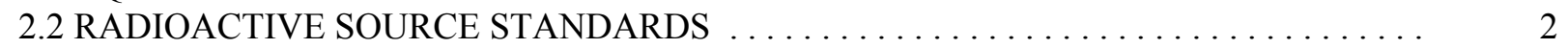

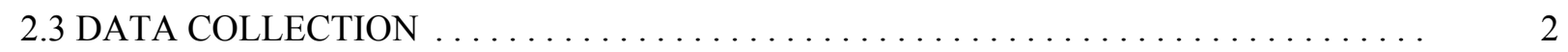

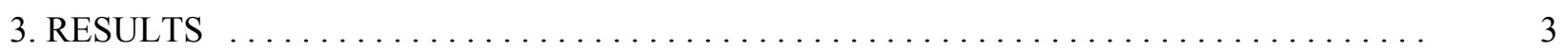

3.1 DETECTOR PERFORMANCE MONITORING STANDARD SOURCES $\ldots \ldots \ldots \ldots \ldots$

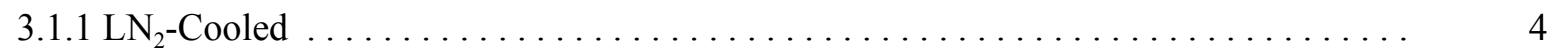

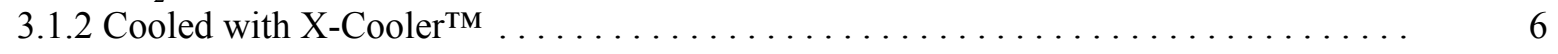

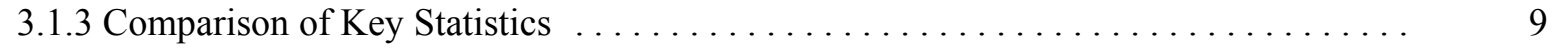

3.2 DETECTOR PERFORMANCE MONITORING BACKGROUND AND COOLED
WITH X-COOLERTM $\ldots \ldots \ldots \ldots \ldots \ldots \ldots \ldots \ldots \ldots \ldots \ldots \ldots \ldots \ldots \ldots \ldots \ldots$

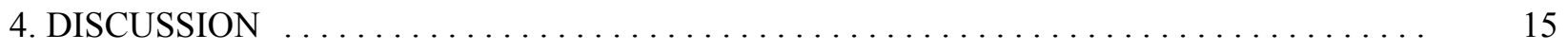

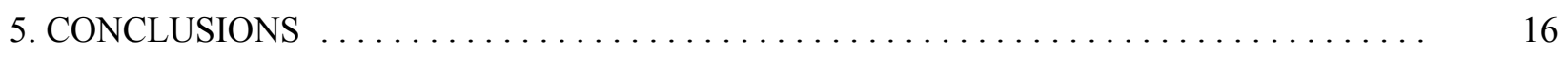

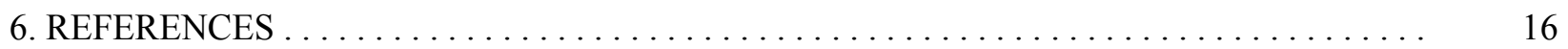

7. APPENDIX A. C++ CODE FOR EXTRACTING AND STORING SPECTRAL DATA . . . 17 



\section{LIST OF FIGURES}

Figure

Page

1 Logic sequence for automated data collection $\ldots \ldots \ldots \ldots \ldots \ldots \ldots \ldots \ldots \ldots \ldots \ldots \ldots \ldots$

2 Peak areas (counts) in regions of interest for safeguards detector SG1 cooled with $\mathrm{LN}_{2}$ and monitoring a standard radioactive source $\ldots \ldots \ldots \ldots \ldots \ldots \ldots \ldots \ldots \ldots \ldots \ldots \ldots \ldots \ldots \ldots$

3 Peak areas (counts) in regions of interest for safeguards detector SG1 cooled using the $\mathrm{X}-$ Cooler $^{\mathrm{TM}}$ and monitoring a standard radioactive source $\ldots \ldots \ldots \ldots \ldots \ldots \ldots \ldots$

4 Distributions of net counts in ROIs from detector SG1 monitoring standard sources and cooled by $\mathrm{LN}_{2}$ (shaded bars) and the X-Cooler ${ }^{\mathrm{TM}}$ (solid bars) . . . . . . . . . . . . . . .

5 Comparison of the distributions of full width at half-maximum for detector SG1 monitoring standard radiation sources and cooled with LN2 and the X-Cooler ${ }^{\mathrm{TM}}$ in four

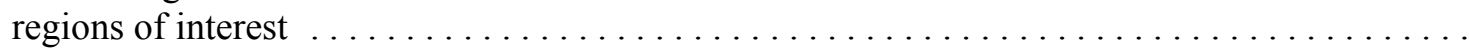

6 Background counts from SG1 cooled by the X-Cooler ${ }^{\mathrm{TM}}$ recorded during consecutive 1 -h live-time intervals in four energy regions of interest

7 Total background counts (a) in consecutive 1-h live-time intervals and (b) distribution of counts for SG1, cooled by the X-Cooler ${ }^{\mathrm{TM}}$, in the 659 - to $664-\mathrm{keV}$ ROI . . . . . . . . . . .

8 Total background counts (a) in consecutive 1-h live-time intervals and (b) distribution of counts for SG1, cooled by the X-Cooler ${ }^{\mathrm{TM}}$, in the 1170 - to $1176-\mathrm{keV}$ ROI . . . . . . . . . . .

9 Total background counts (a) in consecutive 1-h live-time intervals and (b) distribution of counts for SG1, cooled by the X-Cooler ${ }^{\mathrm{TM}}$, in the $1329-$ to $1336-\mathrm{keV}$ ROI . . . . . . . . . . 



\section{LIST OF TABLES}

Table

Page

1 Concentrations of radionuclides with principal emissions used for spectral

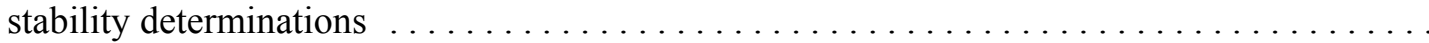

2 Photopeak area statistics for detector SG1 cooled with $\mathrm{LN}_{2}$ and monitoring

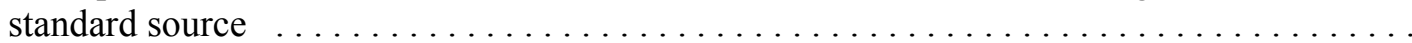

3 Photopeak FWHM statistics for detector SG1 cooled with $\mathrm{LN}_{2}$ and monitoring

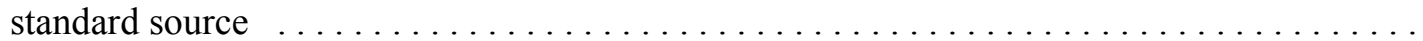

4 Photopeak FWTM statistics for detector SG1 cooled with $\mathrm{LN}_{2}$ and monitoring

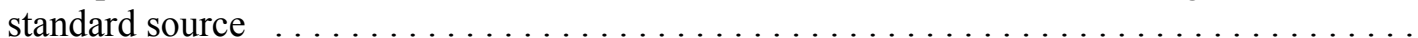

5 Photopeak FW(1/25)M statistics for detector SG1 cooled with $\mathrm{LN}_{2}$ and monitoring

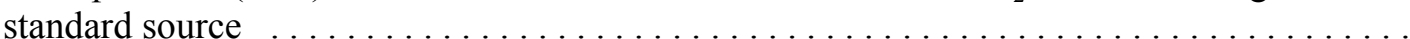

6 Photopeak area statistics for detector SG1 cooled by the X-Cooler ${ }^{\mathrm{TM}}$ and monitoring

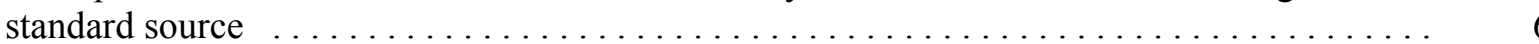

7 Photopeak FWHM statistics for detector SG1 cooled with the X-Cooler ${ }^{\mathrm{TM}}$ and monitoring

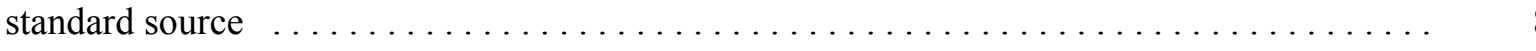

8 Photopeak FWTM statistics for detector SG1 cooled with the X-Cooler ${ }^{\mathrm{TM}}$ and monitoring

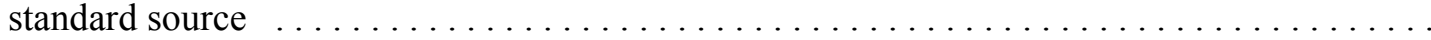

9 Photopeak FW(1/25)M statistics for detector SG1 cooled with the X-Cooler ${ }^{\mathrm{TM}}$ and

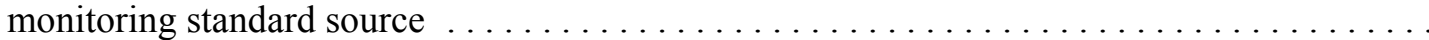

10 Photopeak area statistics for detector SG1 cooled by the X-Cooler ${ }^{\mathrm{TM}}$ and monitoring background 



\section{ACKNOWLEDGMENTS}

The authors wish to acknowledge the generous support of Dr. David P. Spears, Radiation Detection Technologies Program Manager, at the U.S. Department of Energy. Daniel L. Upp, ORTEC ${ }^{\circledR}$ Vice President, was our liaison with PerkinElmer ${ }^{\mathrm{TM}}$ Instruments/ORTEC ${ }^{\circledR}$ Products and provided assistance during $\mathrm{X}-\mathrm{Cooler}^{\mathrm{TM}}$ and germanium detector system procurement, installation and troubleshooting phases of the project. 



\section{ACRONYMS}

$\begin{array}{ll}\text { CV } & \text { Coefficient of variation } \\ \text { DOE } & \text { U.S. Department of Energy } \\ \text { FWHM } & \text { Full width at half-maximum } \\ \text { FWTM } & \text { Full width at tenth-maximum } \\ \text { FWxM } & \text { Full width at } x \text {-maximum } \\ \text { FW(1/25)M } & \text { Full width at } 1 / 25 \text { th maximum } \\ \text { HPGe } & \text { High-purity germanium } \\ \text { LN } & \text { Liquid nitrogen } \\ \text { NIST } & \text { National Institute of Standards and Technology } \\ \text { ROI } & \text { Region of interest } \\ t & \text { Time }\end{array}$





\section{EXECUTIVE SUMMARY}

The ORTEC ${ }^{\circledR}$ X-Cooler ${ }^{\mathrm{TM}}$, because of its potential for unattended or covert use, for use in remote or inaccessible locations, and other advantages over the use of liquid nitrogen $\left(\mathrm{LN}_{2}\right)$, merits consideration as a viable alternative to $\mathrm{LN}_{2}$ for cooling high-purity germanium (HPGe) detectors in many applications. Any decision to use alternative cooling for safeguards monitoring applications must also consider effects on performance characteristics such as total photopeak area, peak area distribution, full width at $x$-maximum $(\mathrm{FW} x \mathrm{M}), \mathrm{FW} x \mathrm{M}$ distribution, and susceptibility to background artifacts, particularly at lower photon energies important in identifying and quantifying special nuclear materials. These parameters bear directly on data quality and usefulness for the intended purpose. This report investigates the performance characteristics of the X-Cooler ${ }^{\mathrm{TM}}$ both in higher-photon-energy regions (662 kev, $1173 \mathrm{kev}$, and $1332 \mathrm{keV}$ ) typical of ( emitting radionuclides and from a low-energy region $(59.5 \mathrm{keV})$ more typical of characteristic X-rays of uranium and the transuranic elements.

Appropriate decisions relating to the use of the X-Cooler ${ }^{\mathrm{TM}}$ in place of $\mathrm{LN}_{2}$, and effective use of the XCooler ${ }^{\mathrm{TM}}$ when it is the chosen alternative, depend on a complete understanding of its performance characteristics in relation to the alternative. Results reported here are germane to three important aspects of the performance of the X-Cooler ${ }^{\mathrm{TM}}$ used to cool HPGe detectors as compared with the benchmark $\mathrm{LN}_{2}$ : (1) stability and reproducibility, (2) precision and (3) limits of detection in HPGe systems cooled by the two alternatives. Stability and reproducibility are reflected through stochastic (random) and non-stochastic (data trends and anomalies) variability when operating under unchanging conditions for long periods of time. Precision is determined by photopeak width, since narrow, well-defined peaks allow more accurate identification of radionuclides, especially in mixtures. Limits of detection are directly related to background levels and variability, so that low backgrounds with minimal variability provide the best limits of detection.

Use of the X-Cooler ${ }^{\mathrm{TM}}$ compared more favorably with $\mathrm{LN}_{2}$ cooling in the higher-energy ROIs than at lower energies. Comparison of photopeak areas in the monitored regions of interest shows that mean peak areas are nearly the same using the $\mathrm{X}-\mathrm{Cooler}^{\mathrm{TM}}$ and $\mathrm{LN}_{2}$ at $1332 \mathrm{keV}$ and $1173 \mathrm{keV}$ (although slightly lower using the $\mathrm{X}$-Cooler ${ }^{\mathrm{TM}}$ ), but are significantly lower for $\mathrm{X}$-Cooler ${ }^{\mathrm{TM}}$ cooling at $662 \mathrm{keV}$ and $59.5 \mathrm{keV}$. The distribution of peak areas in a given ROI, expressed as the coefficient of variation, is almost twice as great using the X-Cooler ${ }^{\mathrm{TM}}$ than when using $\mathrm{LN}_{2}$ in all four of the ROIs investigated. Smaller photopeak areas and greater variability would translate into lower detection efficiencies and greater variability in estimates of quantities of monitored radioactive material.

Peak widths and their distributions were greater with the $\mathrm{X}$-Cooler ${ }^{\mathrm{TM}}$ than with $\mathrm{LN}_{2}$, with the difference between the two cooling methods become greater at lower photon energies. The mean FWHM with the XCooler ${ }^{\mathrm{TM}}$ was about $2.4 \%$ greater than that using $\mathrm{LN}_{2}$ at $1332 \mathrm{keV}$, increasing to around $17 \%$ greater at $59.5 \mathrm{keV}$. The corresponding coefficients of variation ranged from $65 \%$ greater for the X-Cooler at $1332 \mathrm{keV}$ to $112 \%$ greater at $59.5 \mathrm{keV}$. The data for FWTM and FW(1/25)M indicate similar trends for these performance measures. The resolution of complex spectra, especially at low energies, becomes more difficult as the photopeaks widen.

Background increased significantly with one of the the X-Coolers ${ }^{\mathrm{TM}}$ after about 2.5 days (live time) of operation. Such behavior during safeguards monitoring would result in a corresponding change in the limit of detection, perhaps without of the knowledge of the operator. Microphonics transferred to the detector through the X-Cooler ${ }^{\mathrm{TM}}$ coupling is suspected to have been the cause of this particular increase in background. This speculation is supported by the absence of simultaneously increased background levels in the higher-energy ROIs, but the actual cause was not systematically determined. 



\section{INTRODUCTION}

The use of liquid nitrogen $\left(\mathrm{LN}_{2}\right)$ constitutes the current state of the art in cryogenic cooling for highpurity germanium (HPGe) detectors, which are widely used for ( -ray and characteristic X-ray spectroscopy because of their excellent energy discrimination. Use of $\mathrm{LN}_{2}$ requires a liquid nitrogen supply, cumbersome storage tanks and plumbing, and the frequent attention of personnel to be sure that nitrogen levels are sufficient to maintain the detectors at a sufficiently low operating temperature. Safety hazards also are associated with the use of $\mathrm{LN}_{2}$, both because of the potential for severe frostbite on exposure to skin and because it displaces ambient oxygen when it evaporates in closed spaces.

Existing electromechanical coolers have, until now, been more expensive to procure and maintain than $\mathrm{LN}_{2}$ systems. Performance and reliability have also been serious issues because of microphonic degradation of photon energy peak resolution and cooler failures due to compressor oil becoming entrained in the refrigerant.

This report describes the results of tests of a new HPGe detector cooling technology, the PerkinElmer ORTEC ${ }^{\circledR}$ Products X-Cooler ${ }^{\mathrm{TM}}$ that, according to the manufacturer, significantly reduces the lifetime cost of the cooling system without degradation of the output signal ${ }^{1,2}$. The manufacturer claims to have overcome cost, performance and reliability problems of older-generation electromechanical coolers, but the product has no significant history of use, and this project is the first independent evaluation of its performance for safeguard applications.

Total cost savings for the DOE and other agencies that use HPGe systems extensively for safeguards monitoring is expected to be quite significant if the new electromechanical cooler technology is shown to be reliable and if performance characteristics indicate its usefulness for this application. The technology also promises to make HPGe monitoring, characterization and detection available for unattended or covert operation and in remote or inaccessible locations where the unavailability of $\mathrm{LN}_{2}$ and signal degradation from existing mechanical coolers prevent its use at the present time.

\section{EXPERIMENTAL APPROACH}

The performance of spectrometry systems operated with HPGe detectors cooled using $\mathrm{LN}_{2}$ was compared with the performance of the same systems using the PerkinElmer ORTEC ${ }^{\circledR}$ Products X-Cooler ${ }^{\mathrm{TM}}$ in place of $\mathrm{LN}_{2}$. The HPGe detector tested was a medium-efficiency coaxial detector with an active volume of $\sim 128 \mathrm{~cm}^{3}$. Performance indicators consisted of spectroscopic parameters important in safeguards monitoring applications: spectral peak resolution and stability in several energy regions of interest (ROIs).

Testing was performed both in the presence of radioactive source standards and in their absence (background). Regions of interest chosen for evaluation were 58-61 keV, 659-664 keV, 1170-1176 keV, and 1329-1336 keV, corresponding to principal photon emissions of the radioactive source standards.

\subsection{EQUIPMENT}

An ORTEC model SGD-GEM-25175-P-S high-purity germanium (HPGe) coaxial photon detector (serial number 40-TP21490A), with an active volume of $\sim 128 \mathrm{~cm}^{3}$, was operated at the recommended +3600 - $\mathrm{V}$ bias. High voltage and signal processing were provided by a $\mathrm{DSP}^{\mathrm{EC}}{ }^{\circledR}$ digital (-ray spectrometer ${ }^{*}$ (serial no. 421).

*The $\mathrm{DSP}^{\mathrm{EC}}{ }_{\circledast}$ package includes a high-voltage supply and provides analog-to-digital detector signal conversion, digital signal processing and storage, and data transfer by ethernet connection to a personal computer. 


\subsection{RADIOACTIVE SOURCE STANDARDS}

Radioactive source standards were fabricated from a mixture of radionuclides in aqueous solution that were obtained from Analytics (Atlanta, Georgia) and were traceable to the National Institute of Standards and Technology (NIST). The $5.51137 \mathrm{~g}$ of 4-M HCl solution included the radionuclides with principal photopeaks used in this research having total photon fluence rates as indicated in the table below. Individual standards were prepared from the standard solution by evaporation of a weighed aliquot onto an 0.0175 -inch-

Table 1. Concentrations of radionuclides with principal emissions used for spectral stability determinations

\begin{tabular}{cccc}
\hline Radionuclide & $\begin{array}{c}\text { Photopeak } \\
\text { Energy }(\mathrm{keV})\end{array}$ & $\begin{array}{c}\text { Half-life } \\
(\mathrm{y})\end{array}$ & $\begin{array}{c}\text { (-Photon } \\
\text { Fluence Rate } \\
\left(\mathrm{s}^{-1}\right)\end{array}$ \\
\hline${ }^{241} \mathrm{Am}$ & 59.54 & 432 & 1980 \\
${ }^{137} \mathrm{Cs}$ & 661.66 & 30.0 & 1861 \\
${ }^{60} \mathrm{Co}$ & 1173.24 & 5.27 & 3460 \\
${ }^{60} \mathrm{Co}$ & 1332.5 & 5.27 & 3467 \\
\hline
\end{tabular}

${ }^{a}$ Total uncertainty in the photon fluence rate is $\# 5 \%$; the fluence rate was determined on 01 October 2000.

thick (0.444-mm) aluminum planchette. Three individual standards were fabricated using $0.5538 \mathrm{~g}, 0.2956 \mathrm{~g}$ and $0.2988 \mathrm{~g}$ of standard solution, respectively.

\subsection{DATA COLLECTION}

Data collection and analysis methods were automated for the majority of the research. Digital multichannel analyzers were controlled directly using scripts within ORTEC ${ }^{\circledR}$ GammaVision $^{\mathrm{TM}}$ software, while analysis results were extracted and stored using specialized code written in $\mathrm{C}++$. Figure 1 shows a simplified overview of the logic used for the automation sequence.

Each detector was first initiated by clearing the analog-to-digital converter (ADC) memory and setting the acquisition parameters such as 'acquire time'. A spectrum collection cycle would then begin and, once completed, the entire spectrum would be transferred from the ADC to the computer for manipulation. The spectrum would then be analyzed for peaks specific to the study followed by fine-tuning adjustments to the calibration parameters. Data would be extracted and stored into a comma-delimited file using a format similar to the following:

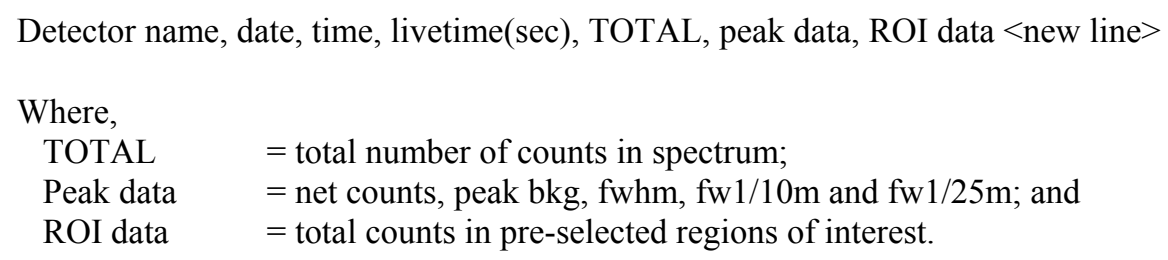


Once initiated, this algorithm was typically allowed to run for extended periods of time without need for extensive manual intervention. Appendix A contains the $\mathrm{C}++$ source code written specifically for extracting and storing spectral information from the $\mathrm{DSP}^{\mathrm{EC}}{ }^{\circledR}$ and GammaVision ${ }^{\mathrm{TM}}$ files. Libraries marketed by the hardware manufacturer were found to be inadequate for the tasks needed in support of this research hence the reason for developing task-specific code. Note that this code does not require the use of any specialized libraries such as those sold by the hardware manufacturer.

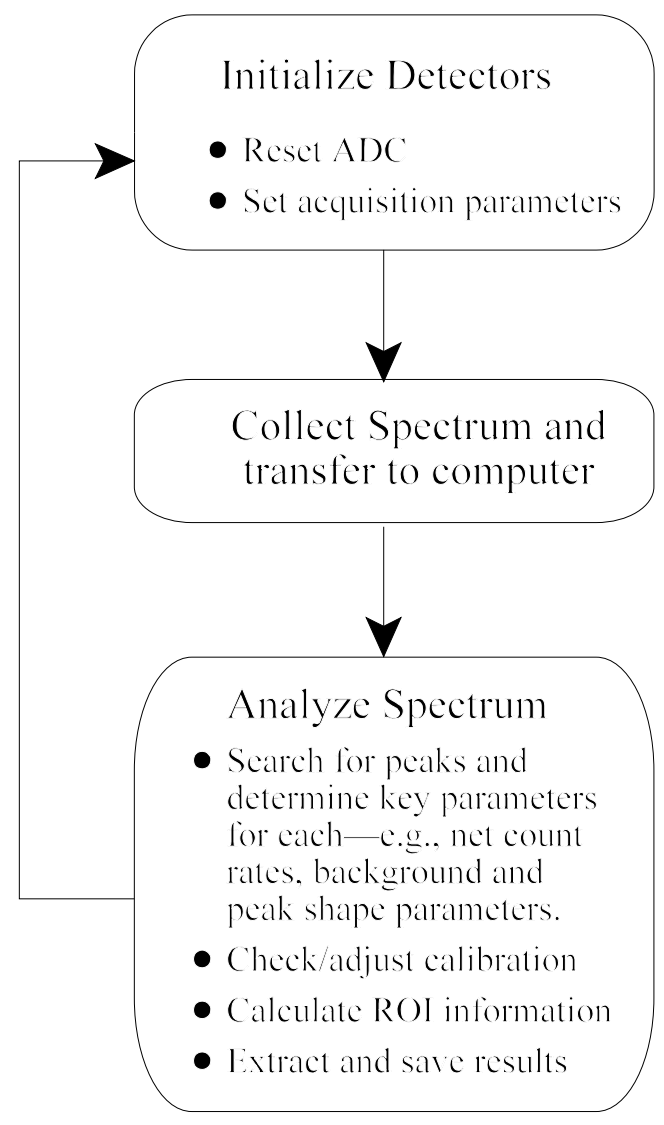

Fig. 1. Logic sequence for automated data collection.

\section{RESULTS}

Recorded data were analyzed for information about the reproducibility and stability of the HPGe system for long periods of time ( $t>500 \mathrm{~h}$, detector live time), both when $\mathrm{LN}_{2}$ cooled and when cooled using the XCooler ${ }^{\mathrm{TM}}$. Sample means and uncertainties were determined for total counts in the ROI (photopeak area), which are adjusted to account both for radioactive decay and detector dead time. Means and uncertainties were also determined for the full photopeak width at half-maximum (FWHM), tenth-maximum(FWTM) and $1 / 25$ th maximum $[\mathrm{FW}(1 / 25) \mathrm{M}]$. The coefficient of variation $(\mathrm{CV})$, or ratio of standard deviation to the mean expressed as a percent, is reported here as a statistic for comparing data sets. The data were also examined for trends and anomalies. 


\subsection{DETECTOR PERFORMANCE MONITORING STANDARD SOURCES}

The coaxial HPGe safeguards detector SG1 was used to collect photon spectral data from standard radioactive sources while cooled with $\mathrm{LN}_{2}$ and again while cooled using the $\mathrm{X}$-Cooler ${ }^{\mathrm{TM}}$. Performance measures are described and compared in the following sections.

\subsection{1 $\mathrm{LN}_{2}$ Cooled}

The spectroscopy system utilizing detector SG1 and monitoring a standard radioactive source was operated for $602 \mathrm{~h}$ with $\mathrm{LN}_{2}$ cooling. Summary statistics for peak areas (total counts) in the ROIs are provided in Table 2. Uncertainty ( \pm 1 standard deviation) in the total counts for each photopeak was less than $1.3 \%$

Table 2. Photopeak area statistics for detector SG-1 cooled with LN2 and monitoring standard source

\begin{tabular}{lcccc}
\hline ROI centroid (keV) & 59.5 & 662 & 1173 & 1332 \\
\cline { 2 - 5 } Number of trials & 602 & 602 & 602 & 602 \\
Mean (counts) & 20209 & 11615 & 10634 & 9478 \\
Maximum (counts) & 21012 & 12266 & 10981 & 9869 \\
Minimum (counts) & 19381 & 11168 & 10025 & 9178 \\
Standard deviation (counts) & 223 & 146 & 124 & 107 \\
CV & $1.10 \%$ & $1.26 \%$ & $1.16 \%$ & $1.13 \%$ \\
\hline
\end{tabular}

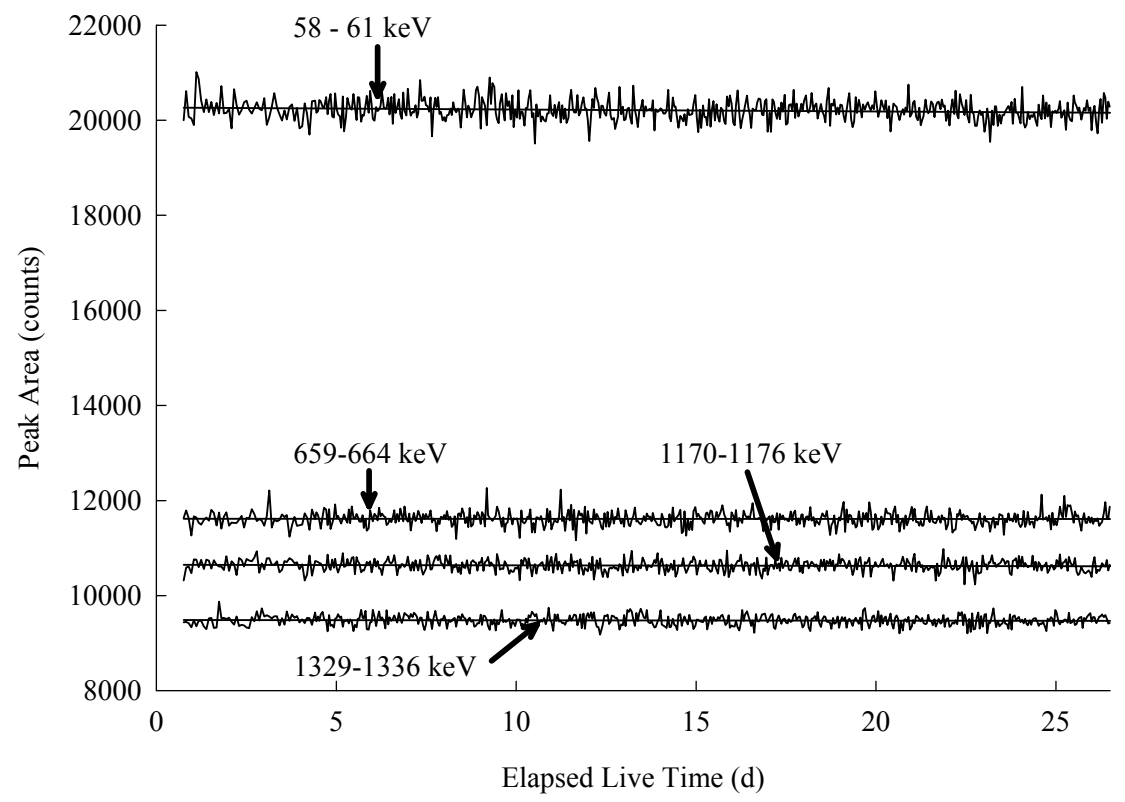

Fig. 2. Peak areas (counts) in regions of interest for safeguards detector SG1 cooled with $\mathrm{LN}_{2}$ and monitoring a standard radioactive source. 
over the test period. Maximum and minimum values were within $6 \%$ of the means. Figure 2 shows peak areas in the ROIs as a function of time. Linear regressions of the data (trend lines in the figure) show that the average detector response was stable over the long monitoring period with slopes of $-4.07 \mathrm{~d}^{-1}(59.5 \mathrm{keV})$, $0.17 \mathrm{~d}^{-1}(662 \mathrm{keV}),-1.15 \mathrm{~d}^{-1}(1173 \mathrm{keV})$, and $-0.79 \mathrm{~d}^{-1}(1332 \mathrm{keV})$, corresponding to changes in average peak areas of the ROIs that are within $-0.70 \%$ of the starting average over the 602 -h counting interval. No periodic features were noted in the data.

Photopeak widths and related statistics for detector SG-1 monitoring standard sources with $\mathrm{LN}_{2}$ cooling are shown in Tables 3 - 5 below. The ratio of FWHM to the peak centroid energy (Table 3 ) was about 1.3\% at $59.5 \mathrm{keV}$ and less than $0.2 \%$ at $662 \mathrm{keV}, 1173 \mathrm{keV}$ and $1332 \mathrm{keV}$. Coefficients of variation for all the peak widths was less than $5 \%$. Tables 4 and 5 provide corresponding statistics for the full width at tenth maximum and full width at $1 / 25$ th maximum.

Table 3. Photopeak FWHM statistics for detector SG1 cooled with LN2 and monitoring standard source

\begin{tabular}{lcccc}
\hline ROI centroid (keV) & 59.5 & 662 & 1173 & 1332 \\
\cline { 2 - 5 } Number of trials & 602 & 602 & 602 & 602 \\
Mean (keV) & 0.763 & 1.317 & 1.640 & $1.732^{\mathrm{a}}$ \\
Maximum (keV) & 0.881 & 1.463 & 1.749 & 1.878 \\
Minimum (keV) & 0.544 & 1.106 & 1.420 & 1.459 \\
Standard deviation (keV) & 0.032 & 0.041 & 0.049 & 0.050 \\
CV & $4.17 \%$ & $3.15 \%$ & $2.99 \%$ & $2.91 \%$ \\
\hline
\end{tabular}

${ }^{\mathrm{a}} \mathrm{FWHM}$ at $1332 \mathrm{keV}$ claimed by the manufacturer is $1.75 \mathrm{keV}$.

Table 4. Photopeak FWTM statistics for detector SG1 cooled with LN2 and monitoring standard source

\begin{tabular}{lcccc}
\hline ROI centroid (keV) & 59.5 & 662 & 1173 & 1332 \\
\cline { 2 - 5 } Number of trials & 602 & 602 & 602 & 602 \\
Mean (keV) & 1.305 & 2.323 & 2.931 & 3.097 \\
Maximum (keV) & 1.438 & 2.513 & 3.085 & 3.664 \\
Minimum (keV) & 1.095 & 2.109 & 2.524 & 3.077 \\
Standard deviation (keV) & 0.035 & 0.046 & 0.051 & 0.049 \\
CV & $2.65 \%$ & $1.96 \%$ & $1.74 \%$ & $1.59 \%$ \\
\hline
\end{tabular}


Table 5. Photopeak FW(1/25)M statistics for detector SG1 cooled with LN2 and monitoring standard source

\begin{tabular}{lcccc}
\hline ROI centroid $(\mathrm{keV})$ & 59.5 & 662 & 1173 & 1332 \\
\cline { 2 - 5 } Number of trials & 602 & 602 & 602 & 602 \\
Mean $(\mathrm{keV})$ & 1.587 & 2.745 & 3.464 & 3.668 \\
Maximum (keV) & 1.694 & 3.050 & 3.664 & 3.863 \\
Minimum (keV) & 1.346 & 2.505 & 3.077 & 3.451 \\
Standard deviation (keV) & 0.034 & 0.059 & 0.063 & 0.065 \\
CV & $2.17 \%$ & $2.14 \%$ & $1.82 \%$ & $1.76 \%$ \\
\hline
\end{tabular}

\subsubsection{Cooled with X-Cooler ${ }^{\mathrm{TM}}$}

The spectroscopy system utilizing detector SG-1 and monitoring a standard radioactive source was operated for $1248 \mathrm{~h}$ (live time) with cooling provided by the X-Cooler ${ }^{\mathrm{TM}}$. Summary statistics for peak areas (total counts) in the ROIs are provided in Table 6. (Fewer than 1248 trials are reported in the table for 1173$\mathrm{keV}$ and $1332-\mathrm{keV}$ centroids because of some excluded anomalous data that were determined to have origins in the data collection and reporting software, and were not due to any factors related to the X-Cooler ${ }^{\mathrm{TM}}$.)

Table 6. Photopeak area statistics for detector SG1 cooled by the $\mathrm{X}$-Cooler ${ }^{\mathrm{TM}}$ and monitoring standard source

\begin{tabular}{lcccc}
\hline ROI centroid (keV) & 59.5 & 662 & 1173 & 1332 \\
\cline { 2 - 5 } Number of trials & 1248 & 1248 & 1246 & 1234 \\
Mean (counts) & 19778 & 11473 & 10583 & 9409 \\
Maximum (counts) & 21220 & 12250 & 11052 & 9783 \\
Minimum (counts) & 15681 & 9564 & 7498 & 7739 \\
Standard deviation (counts) & 397 & 198 & 222 & 180 \\
CV & $2.01 \%$ & $1.73 \%$ & $2.10 \%$ & $1.91 \%$ \\
\hline
\end{tabular}

Uncertainty ( \pm 1 standard deviation) in the total counts for each photopeak was between $1.7 \%$ and $2.1 \%$ of the mean over the test period. Maximum values were within about $7 \%$ of the respective means, but variations in the minimum values were much greater, ranging to almost $30 \%$ below the mean in the case of the 1173 $\mathrm{keV}$ centroid. Figure 3, which shows peak areas in the ROIs as a function of time, reveals that the relatively greater minimum values in Table 6 are the result of several data spikes ( $\sim 1 \mathrm{~h}$ duration) and of one broad dip $(\sim 1 \mathrm{~d})$ in total counts of each of the 4 ROIs. Most of the spikes and the dip are closely related in time. These features are unexplained and cannot necessarily be attributed to the X-Cooler ${ }^{\mathrm{TM}}$ performance. Examination of the figure also reveals a significant drop in counts in the 58- to 61-keV ROI at the end of the test period. This anomaly is also unexplained; there is no obvious corresponding falloff in counts in the other ROIs. Linear regressions of the data (trend lines in the figure) show that the average detector response was otherwise stable over the long monitoring period with slopes of $2.78 \mathrm{~d}^{-1}(59.5 \mathrm{keV}),-0.98 \mathrm{~d}^{-1}(662 \mathrm{keV}),-0.048 \mathrm{~d}^{-1}$ 
$(1173 \mathrm{keV})$, and $-0.31 \mathrm{~d}^{-1}(1332 \mathrm{keV})$, corresponding to changes in average total counts in the ROIs within $-0.45 \%$ to $0.75 \%$ of the starting average over the 1248 -h counting interval. No periodic features were noted in the data.

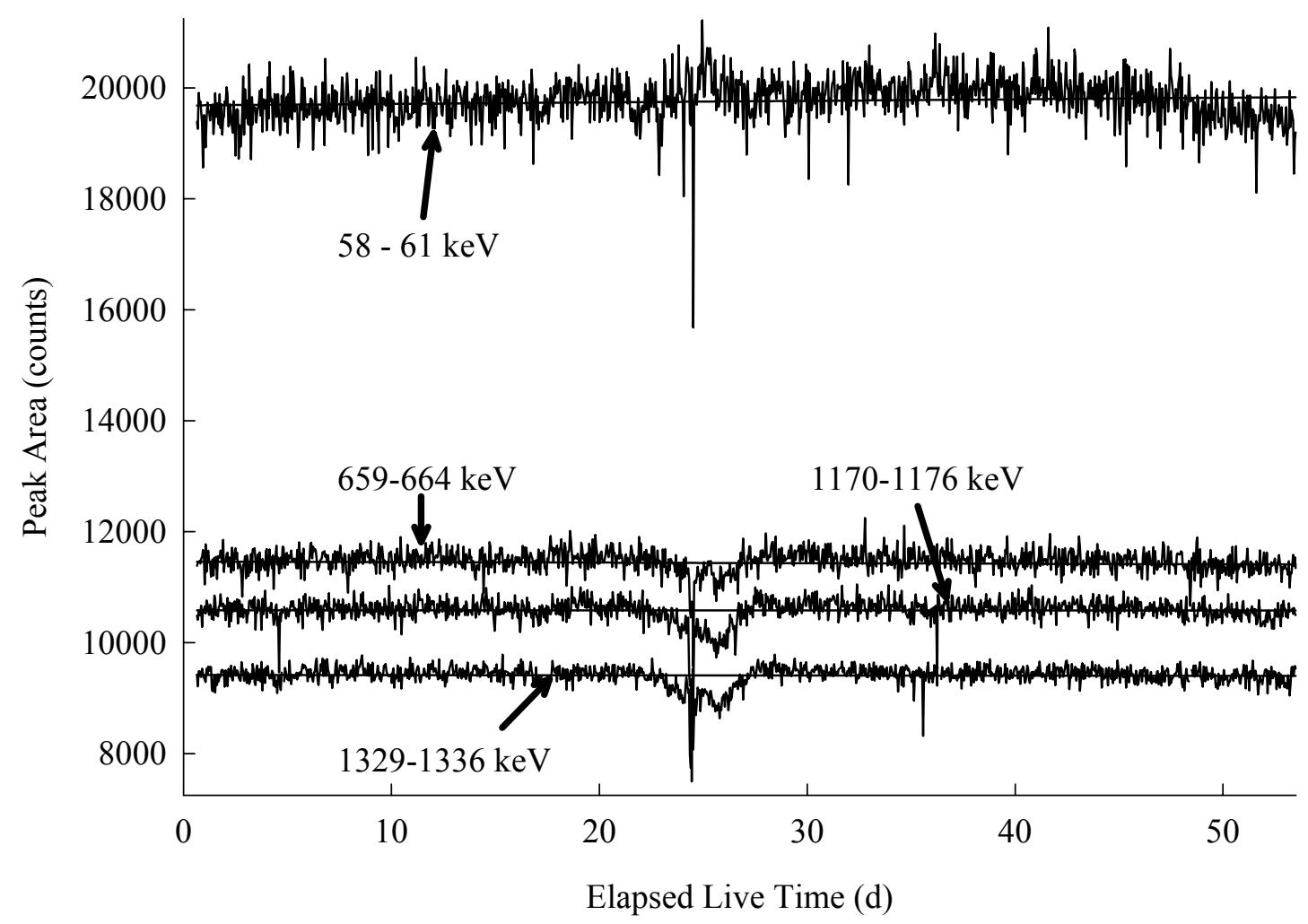

Fig. 3. Peak areas (counts) in regions of interest for safeguards detector SG1 cooled using the $\mathrm{X}-\mathrm{Cooler}^{\mathrm{TM}}$ and monitoring a standard radioactive source.

Photopeak widths and related statistics for detector SG-1 monitoring standard sources and cooled with the X-Cooler ${ }^{\mathrm{TM}}$ are shown in Tables 7 - 9 below. The ratio of FWHM to the peak centroid energy was about $1.5 \%$ at $59.5 \mathrm{keV}$ and about $0.2 \%$ or less at $662 \mathrm{keV}, 1173 \mathrm{keV}$ and $1332 \mathrm{keV}$. Coefficients of variation for the peak widths was between $5.7 \%(1332 \mathrm{keV})$ and about $11.4 \%(59.5 \mathrm{keV})$. Standard deviations in peak widths tended to be relatively constant with centroid energy. Mean peak widths tended to increase with energy, however, so that CVs decreased correspondingly. This behavior is in contrast to that of the detector cooled with LN2 (Tables 3 - 5), where both mean peak widths and their standard deviations increase with centroid energy and CVs stayed about the same or decreased slightly. 
Table 7. Photopeak FWHM statistics for detector SG1 cooled with the $\mathrm{X}$-Cooler ${ }^{\mathrm{TM}}$ and monitoring standard source

\begin{tabular}{lcccc}
\hline ROI centroid $(\mathrm{keV})$ & 59.5 & 662 & 1173 & 1332 \\
\cline { 2 - 5 } Number of trials & 1248 & 1248 & 1246 & 1234 \\
Mean $(\mathrm{keV})$ & 0.921 & 1.412 & 1.718 & $1.798^{\mathrm{a}}$ \\
Maximum $(\mathrm{keV})$ & 1.664 & 2.115 & 2.408 & 2.347 \\
Minimum (keV) & 0.500 & 0.985 & 1.098 & 1.409 \\
Standard deviation $(\mathrm{keV})$ & 0.105 & 0.103 & 0.106 & 0.103 \\
$\mathrm{CV}$ & $11.42 \%$ & $7.33 \%$ & $6.17 \%$ & $5.72 \%$ \\
\hline
\end{tabular}

${ }^{\mathrm{a}} \mathrm{FWHM}$ at $1332 \mathrm{kev}$ claimed by the manufacturer is $1.75 \mathrm{keV}$.

Table 8. Photopeak FWTM statistics for detector SG-1 cooled with the $\mathrm{X}$-Cooler ${ }^{\mathrm{TM}}$ and monitoring standard source

\begin{tabular}{lcccc}
\hline ROI centroid $(\mathrm{keV})$ & 59.5 & 662 & 1173 & 1332 \\
\cline { 2 - 5 } Number of trials & 1248 & 1248 & 1246 & 1234 \\
Mean $(\mathrm{keV})$ & 1.820 & 2.668 & 3.234 & 3.391 \\
Maximum $(\mathrm{keV})$ & 3.109 & 4.338 & 5.964 & 6.117 \\
Minimum (keV) & 1.206 & 1.994 & 2.143 & 2.766 \\
Standard deviation (keV) & 0.171 & 0.160 & 0.172 & 0.162 \\
CV & $9.39 \%$ & $6.00 \%$ & $5.32 \%$ & $4.78 \%$ \\
\hline
\end{tabular}

Table 9. Photopeak FW(1/25)M statistics for detector SG1 cooled with the $\mathrm{X}$-Cooler ${ }^{\mathrm{TM}}$ and monitoring standard source

\begin{tabular}{lcccc}
\hline ROI centroid $(\mathrm{keV})$ & 59.5 & 662 & 1173 & 1332 \\
\cline { 2 - 5 } Number of trials & 1248 & 1248 & 1246 & 1234 \\
Mean $(\mathrm{keV})$ & 2.385 & 3.290 & 3.964 & 4.134 \\
Maximum $(\mathrm{keV})$ & 3.894 & 5.425 & 6.877 & 7.127 \\
Minimum (keV) & 1.535 & 2.499 & 2.666 & 3.347 \\
Standard deviation (keV) & 0.234 & 0.212 & 0.228 & 0.217 \\
CV & $9.81 \%$ & $6.44 \%$ & $5.75 \%$ & $5.25 \%$ \\
\hline
\end{tabular}




\subsubsection{Comparison of Key Statistics}

Figure 4 compares net count distributions for safeguards detector SG1 cooled with $\mathrm{LN}_{2}$ and with the $\mathrm{X}$-Cooler ${ }^{\mathrm{TM}}$. Equal numbers of samples (a sample being a count with live-time duration of $1 \mathrm{~h}$ ) were used for comparison in each ROI. The total dataset (602 samples) from Figure 2 was used as representative of data obtained when using $\mathrm{LN}_{2}$ cooling (shaded bars). A selection of 301 samples from each side of, but excluding, the data anomalies that occurred at around 25 days in Figure 3 (602 samples total), was taken as representative of data in each ROI obtained when using the X-Cooler ${ }^{\mathrm{TM}}$ (solid bars). It is apparent from the figures that distributions from detectors cooled by the X-Cooler ${ }^{\mathrm{TM}}$ are broader, have lower means, and are more likely to contain outliers than those from detectors cooled by $\mathrm{LN}_{2}$, and that the differences between the distributions are more pronounced at lower photon energies.

$59.5 \mathrm{keV}$

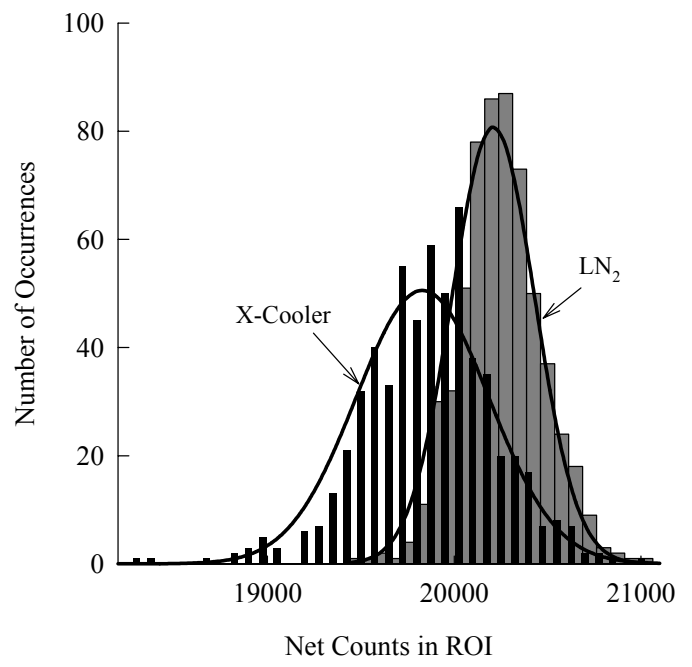

$1173 \mathrm{keV}$

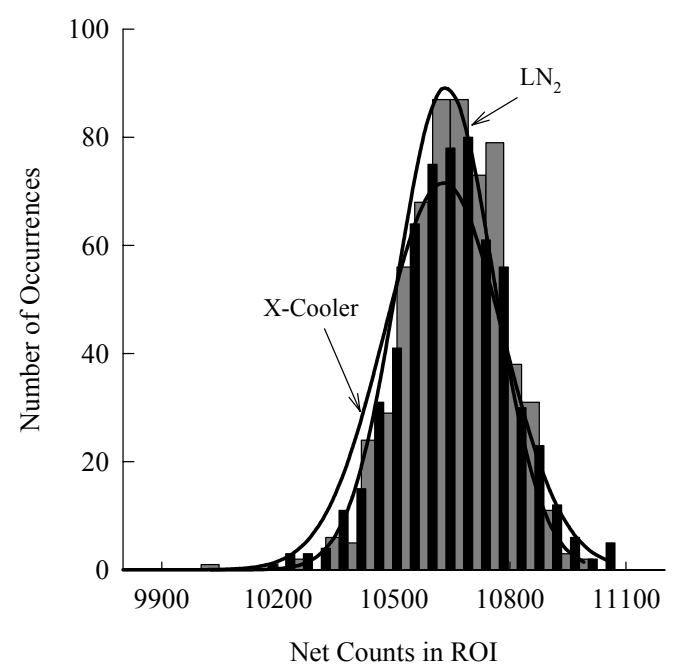

$662 \mathrm{keV}$

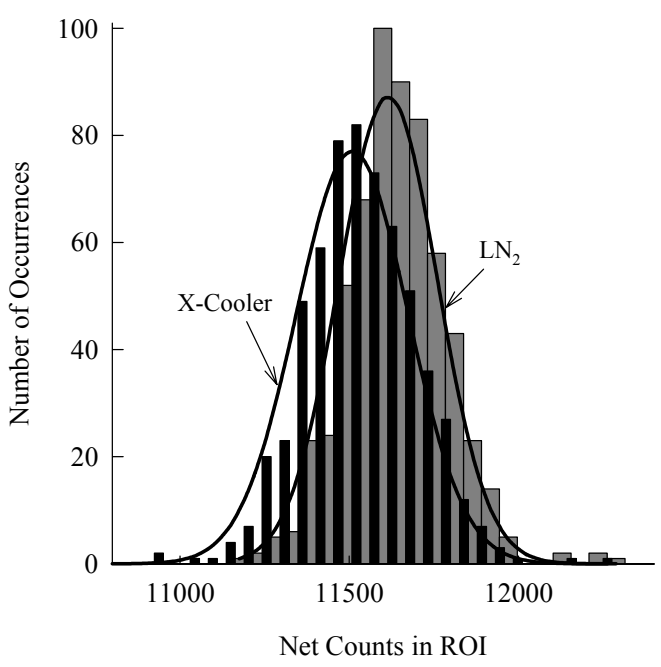

$1332 \mathrm{keV}$

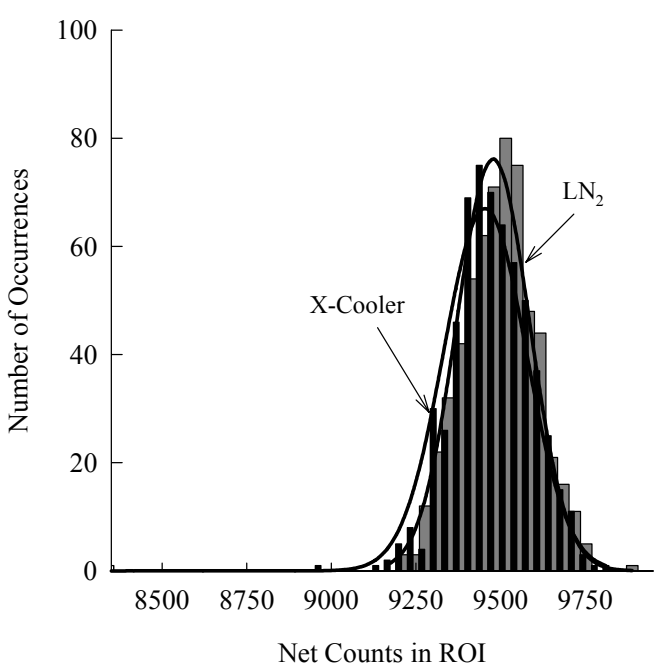

Fig. 4. Distributions of net counts in ROIs from detector SG1 monitoring standard sources and cooled by $\mathrm{LN}_{2}$ (shaded bars) and the $\mathrm{X}$-Cooler ${ }^{\mathrm{TM}}$ (solid bars). 
Distributions of values of FWHM around the centroids of the four ROIs were also determined from the same data sets and are shown in Figure 5. Mean FWHM values were 2\% - 17\% higher for the same detector cooled with the X-Cooler ${ }^{\mathrm{TM}}$, and the standard deviations about the means were $70 \%-148 \%$ larger than when cooled with $\mathrm{LN}_{2}$.

$59.5 \mathrm{keV}$

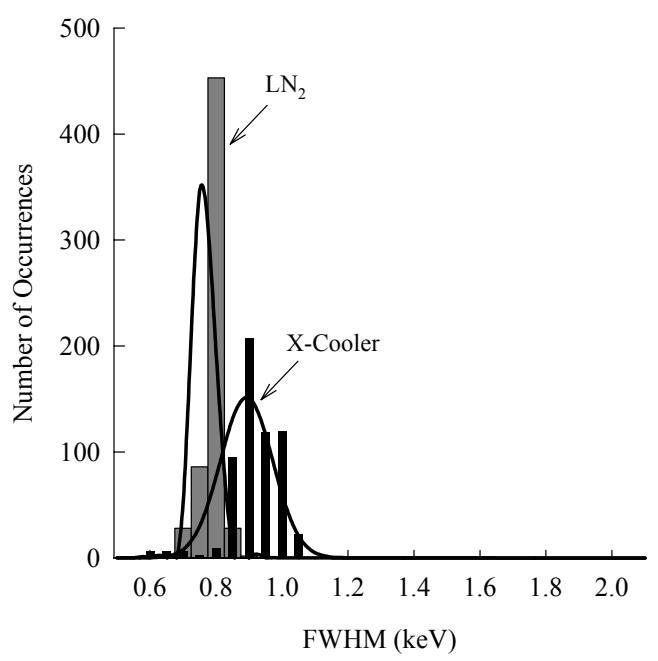

$1173 \mathrm{keV}$

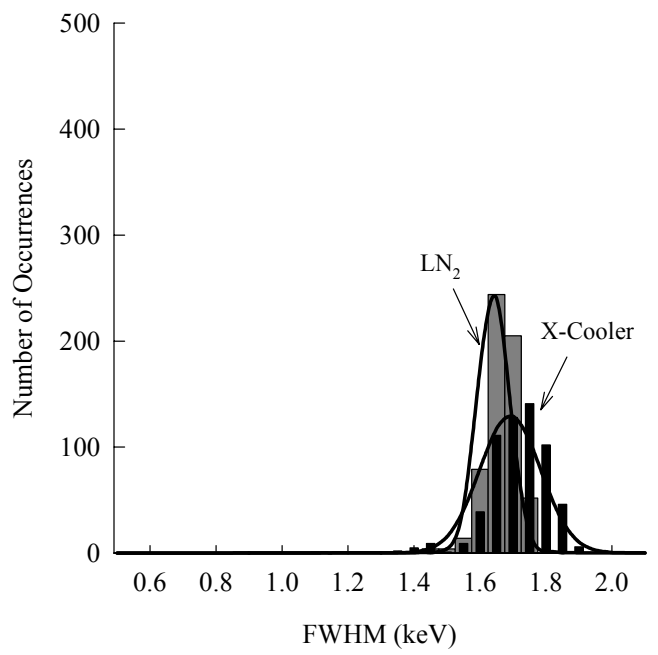

$662 \mathrm{keV}$

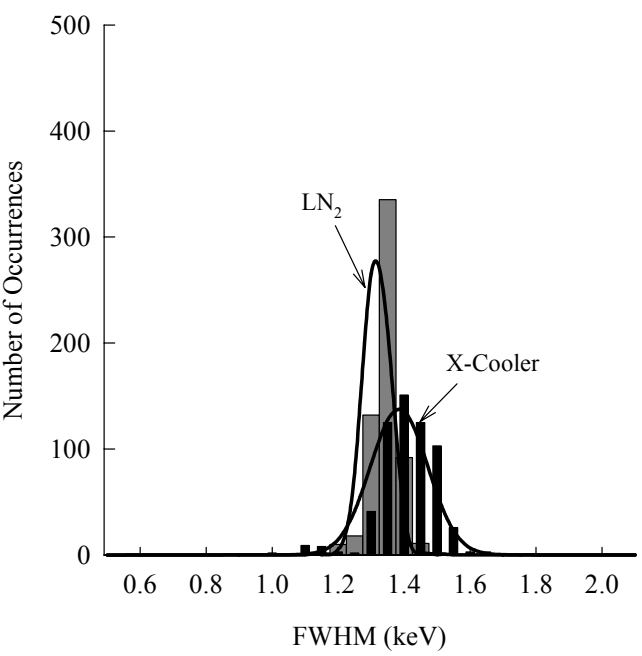

$1332 \mathrm{keV}$

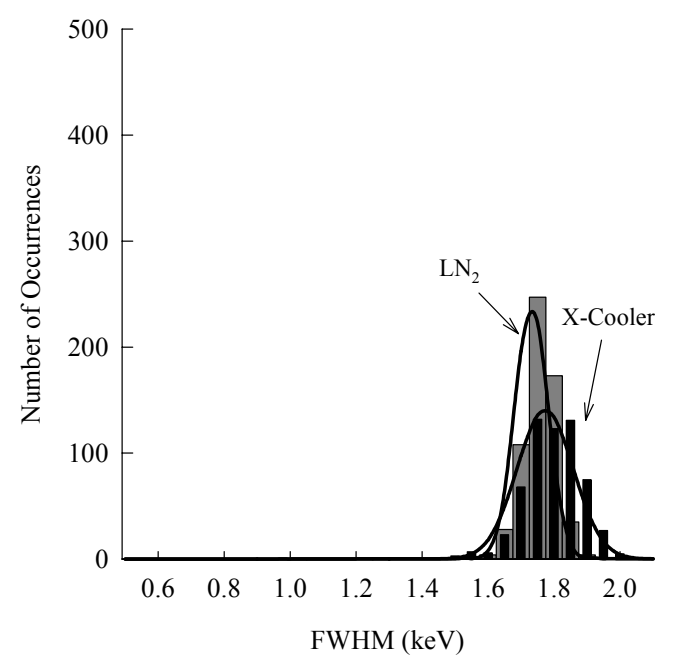

Fig. 5. Comparison of the distributions of full width at half-maximum for detector SG1 monitoring standard radiation sources and cooled with $\mathrm{LN}_{2}$ and the $\mathrm{X}-\mathrm{Cooler}^{\mathrm{TM}}$ in four regions of interest. Shaded bars correspond to the LN2 distributions; solid bars, to those of the X-Cooler ${ }^{\mathrm{TM}}$.

\subsection{DETECTOR PERFORMANCE MONITORING BACKGROUND AND COOLED WITH X-COOLERTM}

The coaxial HPGe safeguards detector SG1 was used to collect background photon spectral data while cooled using the X-Cooler ${ }^{\mathrm{TM}}$. The spectroscopy system utilizing detector SG1 and monitoring background was operated for $187 \mathrm{~h}$ (live time). Summary statistics for peak areas (total counts) in the ROIs are provided in Table 10. Background counts were quite low, with no more than 15 counts in any trial, except in the low- 
energy (59- to 61-keV) region. Examination of Figure 6, which shows the background count rate in consecutive 1-h trials plotted with time, reveals that background response in the low-energy ROI was also low $\left[(26 \pm 9) \mathrm{h}^{-1}\right]$ for about $2 \frac{1}{2} \mathrm{~d}$, after which it gradually increased to around $(325 \pm 125) \mathrm{h}^{-1}$. Replacement of the X-Cooler ${ }^{\mathrm{TM}}$ with another identical X-Cooler ${ }^{\mathrm{TM}}$ eliminated this increase in background. The source of the anomalous behavior in the first X-Cooler ${ }^{\mathrm{TM}}$ was not determined.

Table 10. Photopeak area statistics for detector SG1 cooled by the X-Cooler ${ }^{\mathrm{TM}}$ and monitoring background

\begin{tabular}{lcccc}
\hline ROI (keV) & $58-61$ & $659-664$ & $1170-1176$ & $1329-1336$ \\
\cline { 2 - 5 } Number of trials & 187 & 187 & 187 & 187 \\
Mean (counts) & -- & 6.93 & 3.63 & 3.13 \\
Maximum (counts) & 603 & 15 & 9 & 9 \\
Minimum (counts) & 15 & 2 & 0 & 0 \\
Standard deviation (counts) & -- & 2.38 & 1.87 & 1.79 \\
CV & -- & $34.4 \%$ & $51.6 \%$ & $57.1 \%$ \\
\hline
\end{tabular}

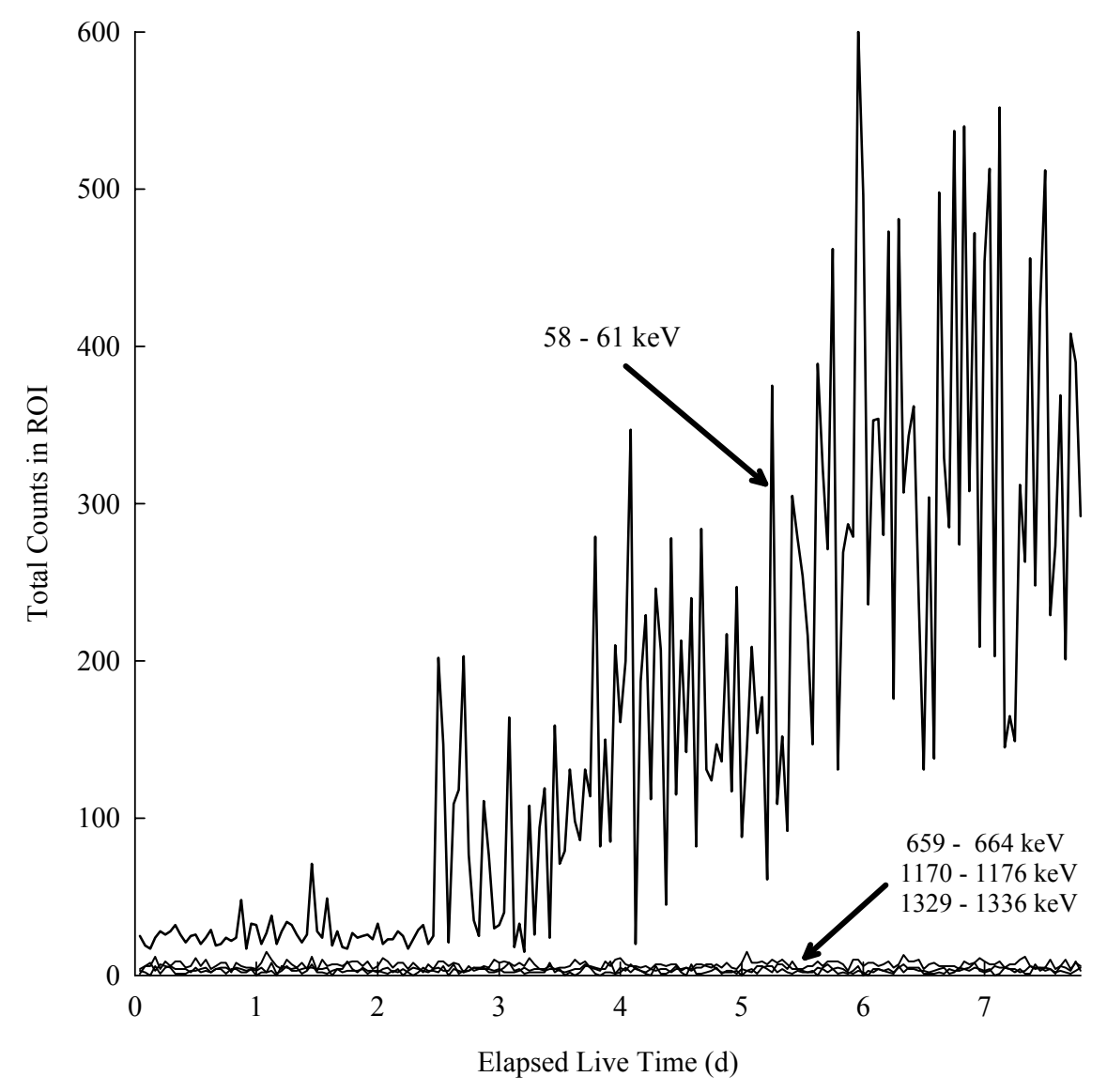

Fig. 6. Background counts from SG1 cooled by the X-CoolerTM recorded during consecutive 1-h live-time intervals in four energy regions of interest. Increased background in the $58-61 \mathrm{keV}$ ROI appears to be due to the onset of microphonic noise from the $\mathrm{X}-\mathrm{Cooler}^{\mathrm{TM}}$. 
Figures 7 through 9 show plots of individual background rates with time and the count rate distributions in 187 trials for ROIs centered on $662 \mathrm{keV}, 1173 \mathrm{keV}$ and $1332 \mathrm{keV}$. The empirical distribution is reasonably represented by a Poisson distribution with the same mean in each case.

(a)

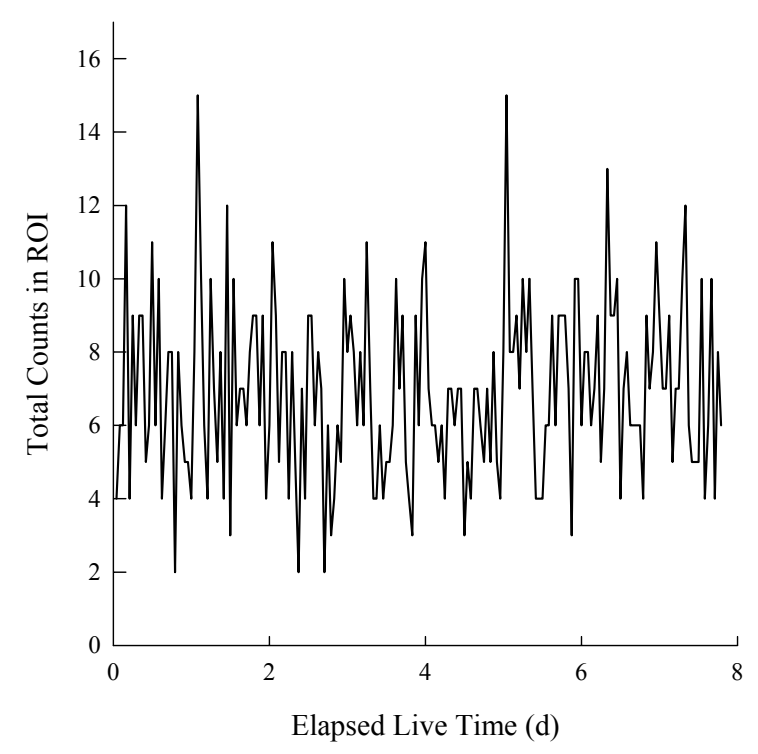

(b)

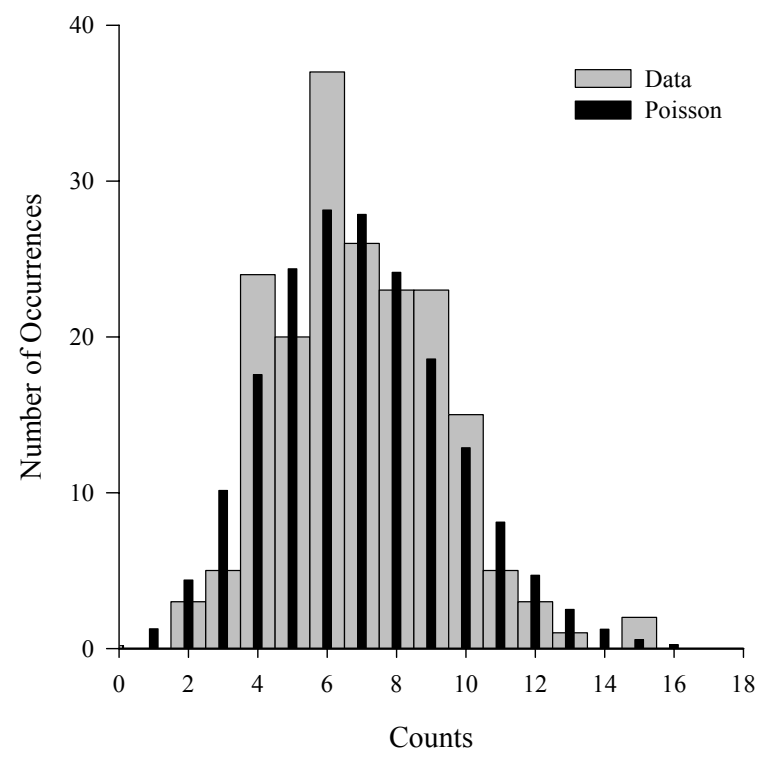

Fig. 7. Total background counts (a) in consecutive 1-h live-time intervals and (b) distribution of counts for SG1, cooled by the $X$ Cooler ${ }^{\mathrm{TM}}$, in the 659- to 664-keV ROI. Solid bars in (b) show the Poisson distribution having the same mean as the data. 
(a)

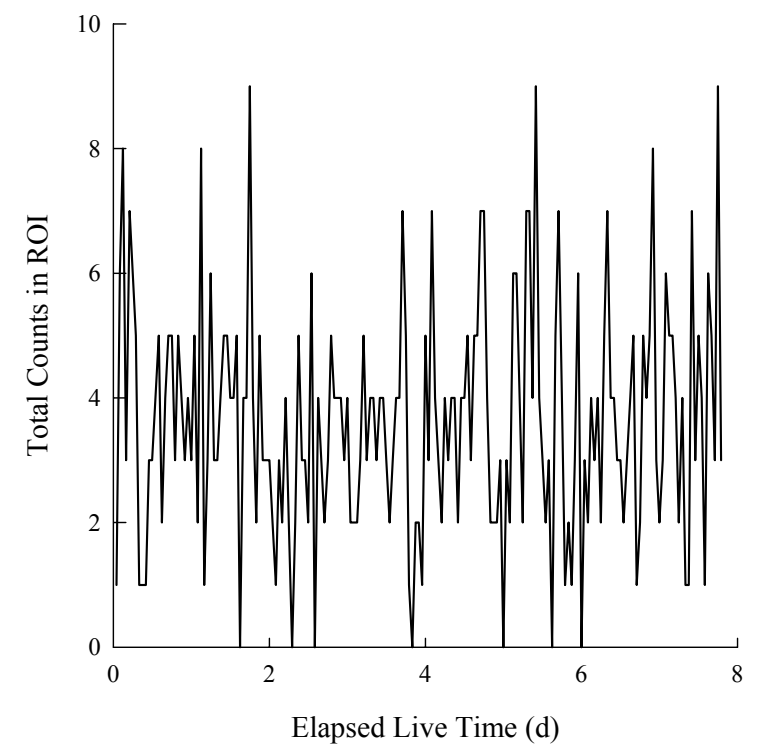

(b)

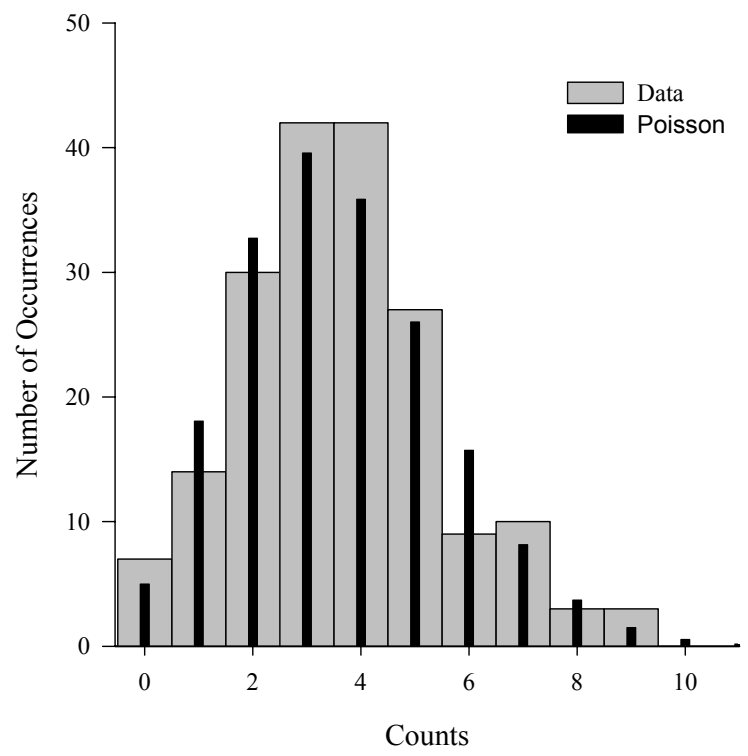

Fig. 8. Total background counts (a) in consecutive 1-h live-time intervals and (b) distribution of counts for SG1, cooled by the XCooler ${ }^{\mathrm{TM}}$, in the 1170- to 1176-keV ROI. Solid bars in (b) show the Poisson distribution having the same mean as the data. 
(a)

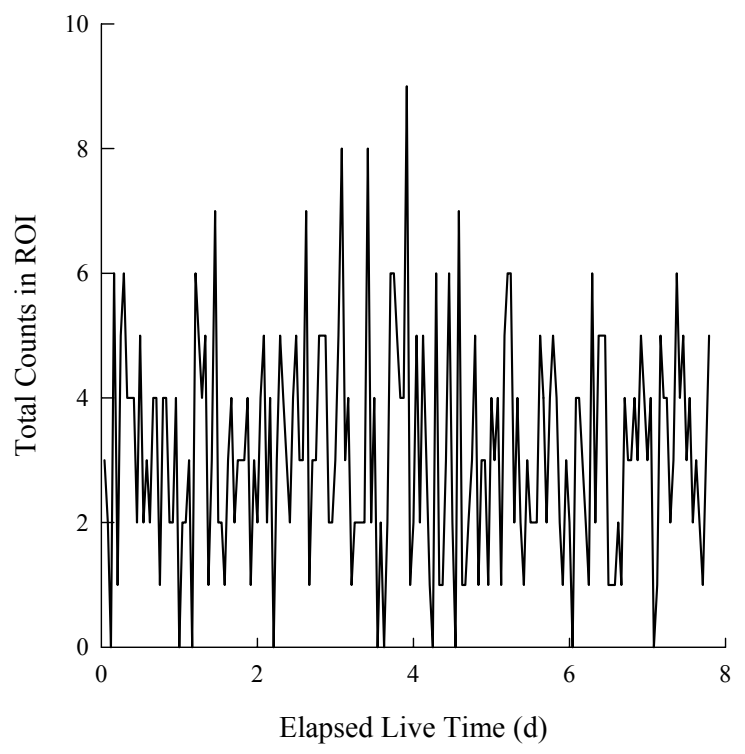

(b)

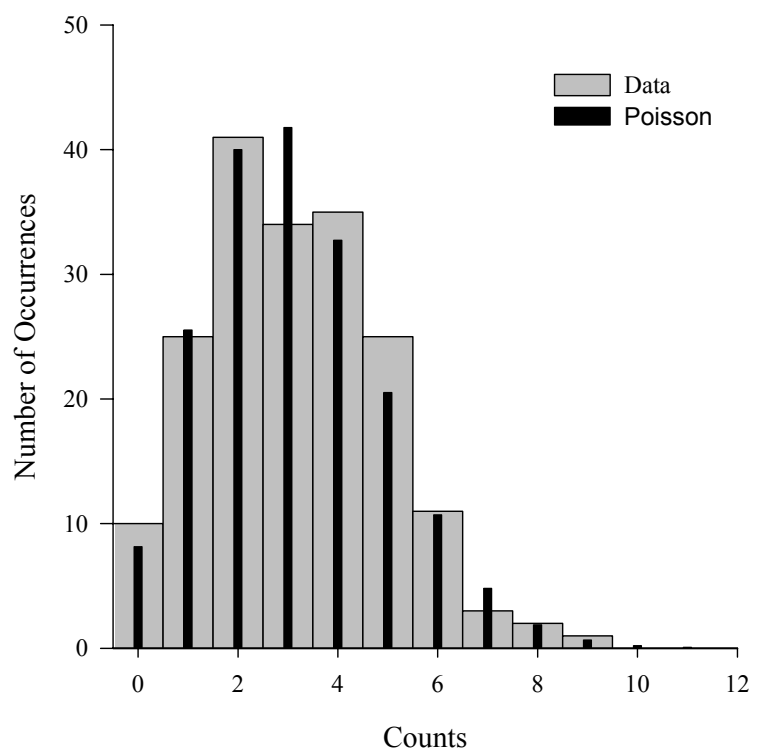

Fig. 9. Total background counts (a) in consecutive 1-h live-time intervals and (b) distribution of counts for SG1 cooled by the XCooler ${ }^{\mathrm{TM}}$, in the 1329- to 1336-keV ROI. Solid bars in (b) show the Poisson distribution having the same mean as the data. 


\section{DISCUSSION}

The X-Cooler ${ }^{\mathrm{TM}}$ was developed as an alternative to $\mathrm{LN}_{2}$ cooling of $\mathrm{HPGe}$ detectors. Among the many potential users of such technology are those involved in nuclear safeguards activities, including special nuclear material monitoring and verification. The data chosen for analysis include both those from higherenergy regions ( $662 \mathrm{kev}, 1173 \mathrm{kev}$, and $1332 \mathrm{keV}$ ) typical of (-emitting radionuclides and from a low-energy region $(59.5 \mathrm{keV})$ more typical of characteristic X-rays of uranium and the transuranic elements and having perhaps greater interest in nuclear safeguards applications.

Appropriate decisions relating to the use of the X-Cooler ${ }^{\mathrm{TM}}$ in place of $\mathrm{LN}_{2}$, and effective use of the XCooler $^{\mathrm{TM}}$ when it is the chosen alternative, depend on a complete understanding of its performance characteristics in relation to the alternative. Results reported here are germane to three important aspects of the performance of the X-Cooler ${ }^{\mathrm{TM}}$ used to cool HPGe detectors as compared with the benchmark $\mathrm{LN}_{2}$ : (1) stability and reproducibility, (2) precision and (3) limits of detection in HPGe systems cooled by the two alternatives. Stability and reproducibility are reflected through stochastic (random) and non-stochastic (data trends and anomalies) variability when operating under unchanging conditions for long periods of time. Precision is determined by photopeak width, since narrow, well-defined peaks allow more accurate identification of radionuclides, especially in mixtures. Limits of detection are directly related to background levels and variability, so that low backgrounds with minimal variability provide the best limits of detection.

Use of the X-CoolerTM compared more favorably with $\mathrm{LN}_{2}$ cooling in the higher-energy ROIs than at lower energies. Comparison of photopeak areas in the monitored regions of interest (Tables 2 and 6, and Figure 4) shows that mean peak areas were nearly the same using the X-Cooler ${ }^{\mathrm{TM}}$ and $\mathrm{LN}_{2}$ at $1332 \mathrm{keV}$ and $1173 \mathrm{keV}$ (although slightly lower using the $\mathrm{X}$-Cooler ${ }^{\mathrm{TM}}$ ), but were significantly lower for $\mathrm{X}$-Cooler ${ }^{\mathrm{TM}}$ cooling at $662 \mathrm{keV}$ and $59.5 \mathrm{keV}$. The distribution of peak area results, expressed as the coefficient of variation, was almost twice as great using the $\mathrm{X}-\mathrm{Cooler}^{\mathrm{TM}}$ than when using $\mathrm{LN}_{2}$ in all four of the ROIs investigated.

Peak widths and their distributions were significantly greater with the $\mathrm{X}-\mathrm{Cooler}^{\mathrm{TM}}$ than with $\mathrm{LN}_{2}$, with the difference between the two cooling methods increasing as photon energy decreases. Figure 5 shows graphically the statistics reported for full width at half-maximum using $\mathrm{LN}_{2}$ (Table 3) and using the XCooler $^{\mathrm{TM}}$ (Table 7, but with anomalous data and trends not believed related to X-Cooler ${ }^{\mathrm{TM}}$ performance removed), illustrating the differences between the two cooling methods. The mean FWHM with the XCooler ${ }^{\mathrm{TM}}$ was about $2.4 \%$ greater than that using $\mathrm{LN}_{2}$ at $1332 \mathrm{keV}$, increasing to around $17 \%$ greater at $59.5 \mathrm{keV}$. The corresponding coefficients of variation ranged from $65 \%$ greater for the $\mathrm{X}$-Cooler at $1332 \mathrm{keV}$ to $112 \%$ greater at $59.5 \mathrm{keV}$. The data for FWTM and FW(1/25)M indicated similar trends for these performance measures.

Increased background from SG1 after about 2.5 days (live time) of operation, shown in Figure 6, raises an additional issue about long-term stability and reproducibility of X-Cooler ${ }^{\mathrm{TM}}$ performance, particularly for safeguards applications. Such behavior during safeguards monitoring would result in a corresponding change in the limit of detection, perhaps without of the knowledge of the operator. Microphonics transferred to the detector through the X-Cooler ${ }^{\mathrm{TM}}$ coupling is suspected to have been the cause of the increased background. This speculation is supported by the absence of simultaneously increased background levels in the higherenergy ROIs, which, we note from past experience, tend to be less susceptible to vibration. The actual cause was not systematically determined, however.

Additional data (including backgrounds collected with LN2 cooling and detailed analysis of X-CoolerTM maintenance and repair history) that would have been helpful in the comparison of X-Cooler ${ }^{\mathrm{TM}}$ and $\mathrm{LN}_{2}$ cooling of HPGe systems are not available for comparison because of early withdrawal of project support. 


\section{CONCLUSIONS}

The X-Cooler ${ }^{\mathrm{TM}}$, because of its potential for use in remote or inaccessible locations and its other advantages over the use of $\mathrm{LN}_{2}$, merits consideration as a viable alternative to $\mathrm{LN}_{2}$ for cooling HPGe detectors in many applications. Its suitability for use in specific safeguards monitoring applications and procedures for its use in such applications should be determined by considering performance characteristics (total peak area, peak area distribution, full width at $x$-maximum, $\mathrm{FW} x \mathrm{M}$ distribution, and susceptibility to background artifacts) at lower photon energies important in identifying and quantifying special nuclear materials. HPGe performance when cooled by the $\mathrm{X}-$ Cooler $^{\mathrm{TM}}$ tended in this study to compare favorably with that when cooled with $\mathrm{LN}_{2}$ at higher photon energies, but less favorably at lower energies. Use of the X-Cooler ${ }^{\mathrm{TM}}$ may be worthy of consideration for many safeguards applications, but any decision for its utilization should be made with cognizance of its limitations and the implications for data quality and usefulness for the intended purpose.

\section{REFERENCES}

1. E. Broerman, R. Keyser, T. Twomey, and D. Upp, "A New Cooler for HPGe Detector Systems", PerkinElmer Instruments-ORTEC. Paper presented at the 23rd meeting of the European Safeguards Research and Development Association on Safeguards and Nuclear Materials Management, Brugge, Belgium, May 2001. Available on the World-Wide Web at http://www.ortec-online.com/papers/reprints.htm.

2. E. Broerman, D. Upp, and T. Twomey, "Performance of a New Type of Electrical Cooler for HPGe Detector Systems." Paper presented at the 42nd Annual Meeting of the Institute of Nuclear Materials Management, Indian Wells CA, July 18, 2001. Available on the World-Wide Web at http://www.ortec-online.com/papers/reprints.htm. 
APPENDIX A

C++ Code for Extracting and Storing Spectral Data 

APPENDIX A. C++ Code for Extracting and Storing Spectral Data

(excludes headers and 'include' files)

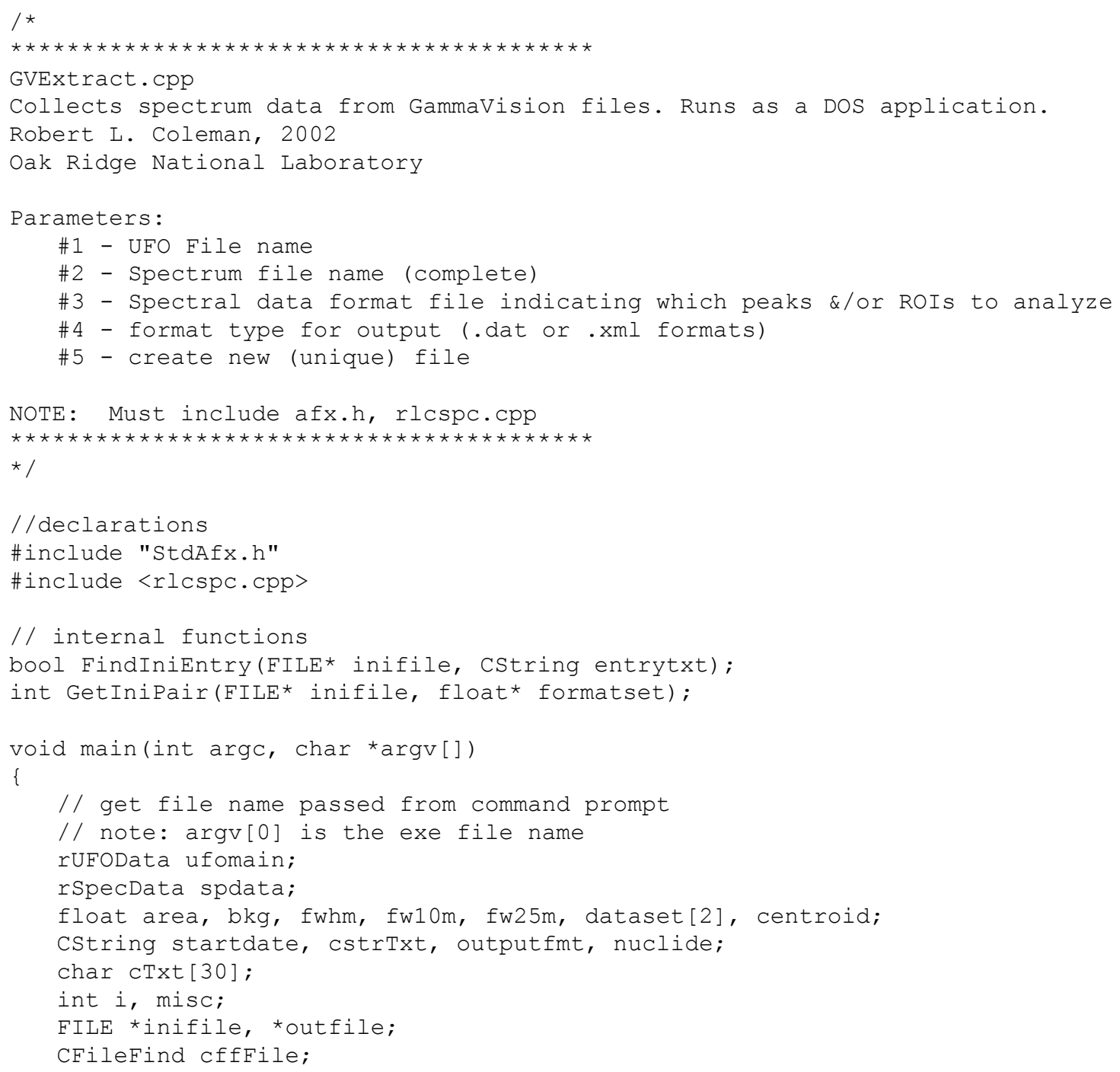




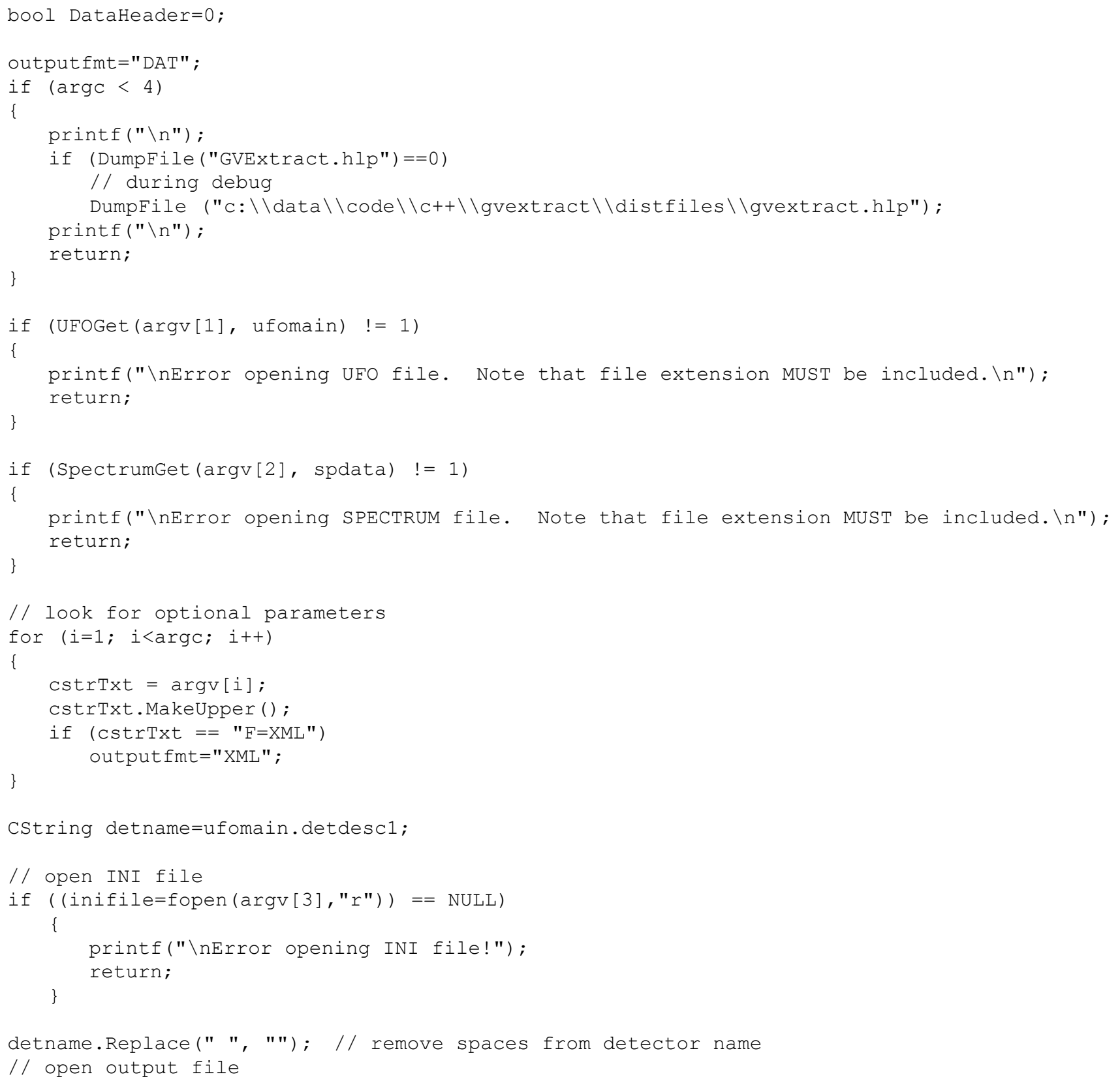

CString detname=ufomain.detdesc1;

// open INI file

if ((inifile=fopen (argv[3],"r")) == NULL) printf("\nError opening INI file!"); return;

detname.Replace(" ", ""); // remove spaces from detector name // open output file 
if (outputfmt $==$ "DAT")

// append to existing file or create new

outfile=fopen (detname+".dat", "a");

else

// create unique file everytime

time_t now;

time (\&now):

outfile=fopen (detname+"_"+_toa (now,cTxt,10)+".xml", "a");

if (fgetc(outfile) $==$ EOF \&\& outputfmt=="XML")

// new file -- output XML header entry

fprintf(outfile,"<?xml version=\"1.0\" ?>\n");

fprintf(outfile,"<!--GammaSpec ROI Measurement Results--> $\backslash n "$ );

// output common data

if (outputfmt $==$ "DAT")

fprintf(outfile," $\% s, \circ s, \circ s, \circ 10.0 f, \circ 5.3 e "$, detname, ufomain.datestr, ufomain.timestr, ufomain.livetime, spdata.sum);

else

// XML output

fprintf (outfile," $<$ SpecResult $>\backslash \mathrm{n} ")$;

fprintf (outfile," $\backslash t<$ Detector $>\% s</$ Detector $>\backslash n "$, detname);

fprintf (outfile," \t<Date $>\circ s<$ Date $>\backslash n "$, ufomain.datestr);

fprintf (outfile," $\backslash t<$ StartTime $>$ o $s<$ StartTime $>\backslash n "$, ufomain.timestr);

fprintf (outfile," $\backslash$ t<LiveTime $>05.3 e</$ LiveTime $>\backslash n "$, ufomain.livetime);

fprintf(outfile," \t<TotalCounts $>\div 5.3 e</$ TotalCounts $>\backslash n "$, spdata.sum);

// extract peak data using FORMAT template file

// get peak data and output to DAT file

if (FindIniEntry(inifile, "[PEAK]"))

while (!feof(inifile))

if $(($ misc=GetIniPair (inifile, dataset $))==1)$

// valid data set retrieved-- output results

if (!DataHeader)

// print opening Peak Data header for XML 
fprintf (outfile, "\t<PeakData $>\backslash \mathrm{n} "$ ) ;

DataHeader=1;

area $=0 ; \mathrm{bkg}=0 ; \mathrm{fwhm}=0 ; \mathrm{fw} 10 \mathrm{~m}=0 ; \quad \mathrm{fw} 25 \mathrm{~m}=0$;

nuclide=""; centroid=dataset [0] ;

if ((i=UFOFindPeak (ufomain, dataset[0], dataset[1])) > -1)

area $=$ ufomain.peak[i].netarea;

$\mathrm{bkg}=$ ufomain.peak[i].background;

$\mathrm{fwhm}=$ ufomain.peak[i].fw2m e;

$\mathrm{fw} 10 \mathrm{~m}=u$ fomain.peak [i]. fw10m_e;

fw25m=ufomain.peak[i].fw25m e;

nuclide=ufomain.peak [i].nuclidename

\} //endif

if (outputfmt $==$ "DAT")

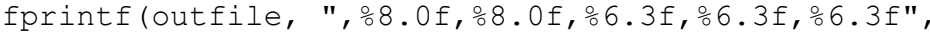

lse

area, bkg, fwhm, fw10m, fw25m);

// XML output

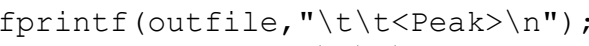

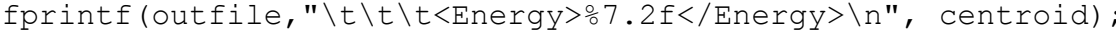

fprintf (outfile," $\backslash t \backslash t \backslash t<$ NetArea $>0.0 \mathrm{f}</$ NetArea $>\backslash \mathrm{n} "$, area)

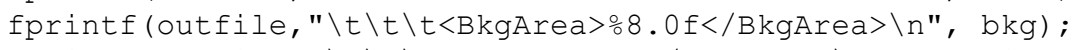

fprintf (outfile," $\backslash t \backslash t \backslash t<$ Isotope $>$ o $<</$ Isotope $>\backslash n "$, nuclide) ;

\}

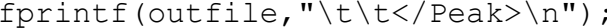

\} //endif

else if $(\mathrm{misc}==0)$

$/ /$ end-of-section or end-of-file

break;

else if $(\operatorname{misc}==-1)$

$/ /$ error in data

printf("Error occurred reading PEAK data from INI file. Press any key.");

keypress ();

return;

\} //end elseif

\} //endwhile

if (outputfmt $==$ "XML")

// print closing section

fprintf (outfile," $\backslash t</$ PeakData $>\backslash n "$ ) ;

\} / /endif 


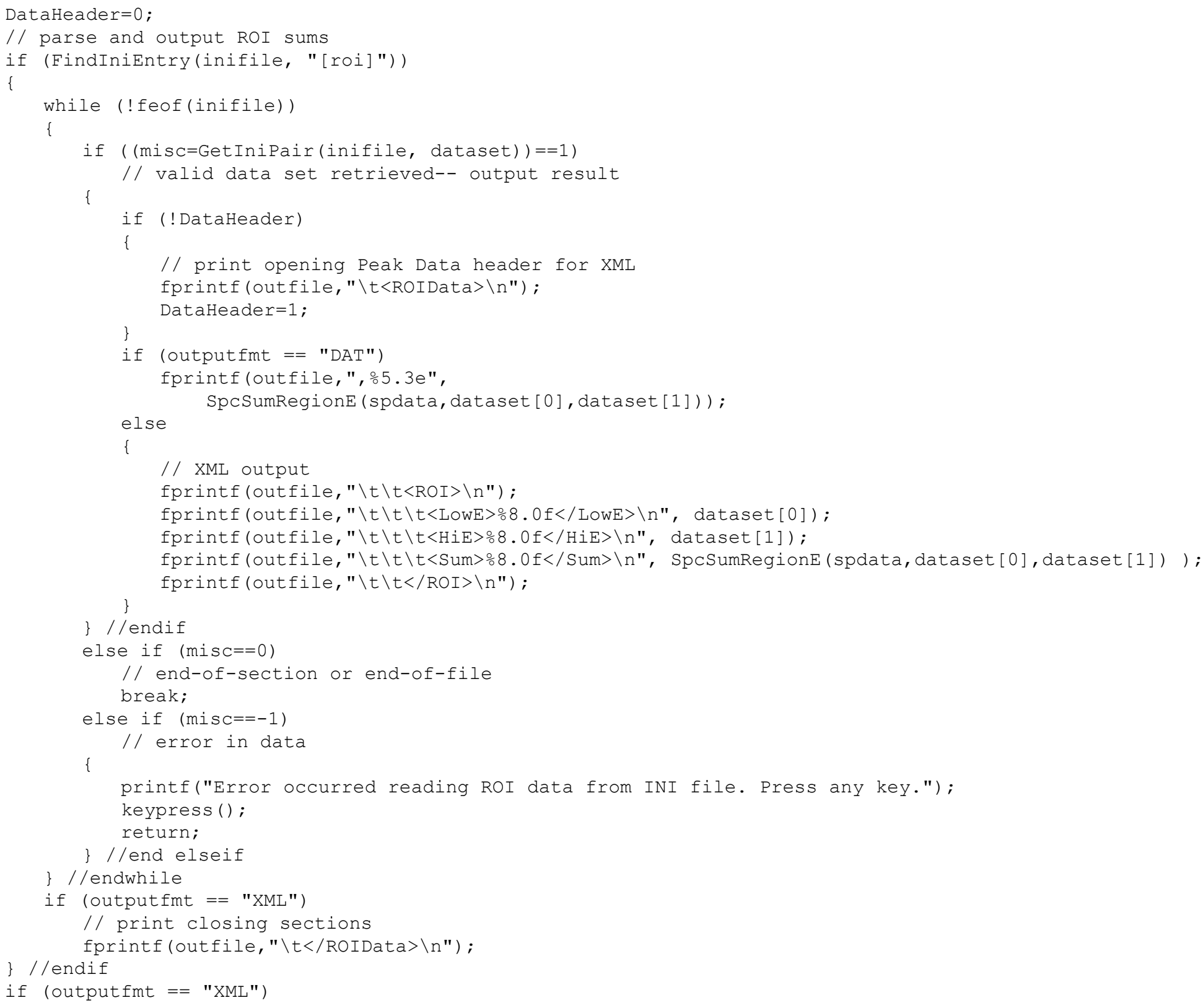

if (outputfmt $==$ "XML") 
// print closing sections

fprintf (outfile,"</SpecResult> \n") ;

fprintf (outfile," $\backslash n "$ ) ;

fclose (outfile);

fclose(inifile);

\} //end main

bool FindIniEntry(FILE* inifile, CString CSentrytxt)

// searches for entrytxt (case insensitive) in file inifile (text file)

// returns 1 if found, 0 if not

fseek(inifile,0,SEEK_SET);

bool rtn=0;

char txt $[200]$

CString cstrTxt;

CSentrytxt.MakeUpper ();

while (!feof(inifile) \&\& rtn==0)

fscanf (inifile, "ㅇs"txt);

cstrTxt=txt;

cstrTxt.MakeUpper();

if (cstrTxt.Find("/") >-1)

fgets (txt,200,inifile);

else if (cstrTxt==CSentrytxt)

rtn $=1$;

\}//endwhile

return rtn;

// dump remainder of line and loop

int GetIniPair(FILE* inifile, float* formatset)

// get a set of format data $\times 1, x 2$ from inifile and store into

// array formatset $[0,1]$

// Return $=0$ if end of file or another section has been reached

$/ /=1$ if VALID data is retrieved

// = - if INVALID data is retrieved

char txt[200], *stopstr;

CString cstrTxt; 


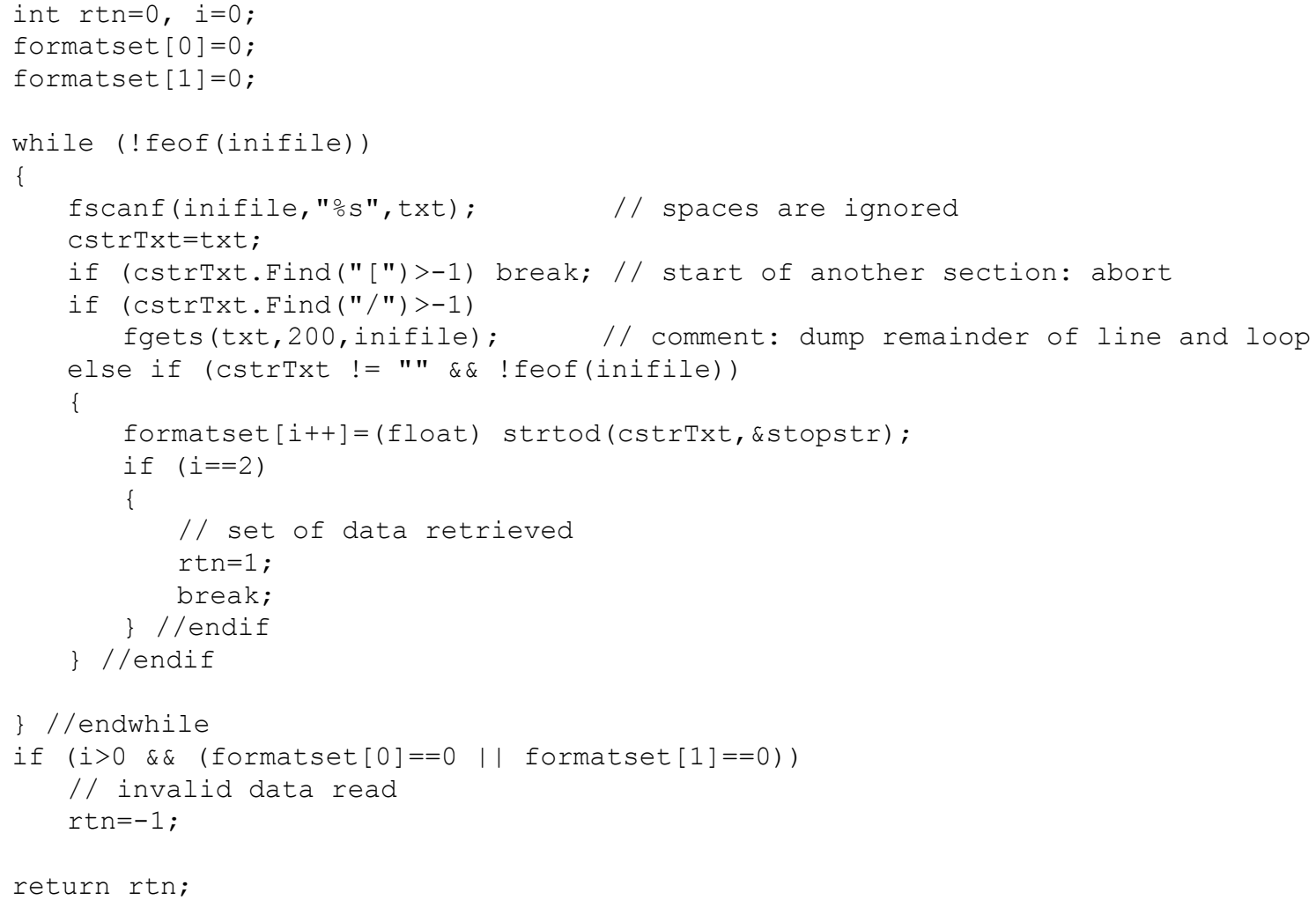

return rtn;

\} 
$/ \star$

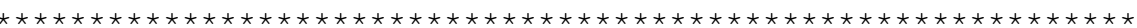

rlcspc.cpp

Functions for accessing SPC, CHN and UFO data

Robert L. Coleman, 2002

Oak Ridge National Laboratory

Notes: 1) Requires the following includes in the project: $\langle a f x . h\rangle,\langle r l c l i b . c p p\rangle$

<afxdisp.h>, <cmath>

Requires compilation using MFC

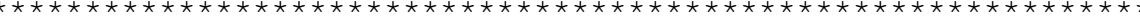

$\star$

\#if ! defined (rlcspc cpp included)

\#define rlcspc_cpp_iñcluded

\#include <rlclib.cpp>

\#define CHN -1

\#define SPC 1

// User-called Functions

int SpectrumGet (char* infilename, struct rspecData \&spdata);

// Opens infilename spectrum file and fills structure spdata

// Works for real or int SPC files and also CHN files.

// Returns -1 if input file does not exist

// 0 if file cannot be a spectra file

1 for success

float SpcSumRegionE (struct rSpecData spectrum, float e1, float e2);

// sums channel counts between el keV and e2 keV of spectrum

int UFOGet (char* infilename, struct rUFOData \&udata);

// Opens infilename UFO file and fills structure udata

ColeDateTime DATEortec(double datetime);

// converts ortec date+time real value to a ColeDateTime DATE value

int UFOFindPeak (rUFOData \&udata, float e1, float e2);

// returns index pointer into peak array contained in udata.

// el is the target peak energy wanted (keV)

// e2 is the tolerance allowed for the peak (keV) 
float ChtoE(float c, float zero, float slope, float poly);

// converts channel c to energy (keV) given al,a2, a3 slope coefficients

int EtoCh(float c, float zero, float slope, float poly);

// converts energy e (keV) to channel given a1,a2,a3 slope coefficients

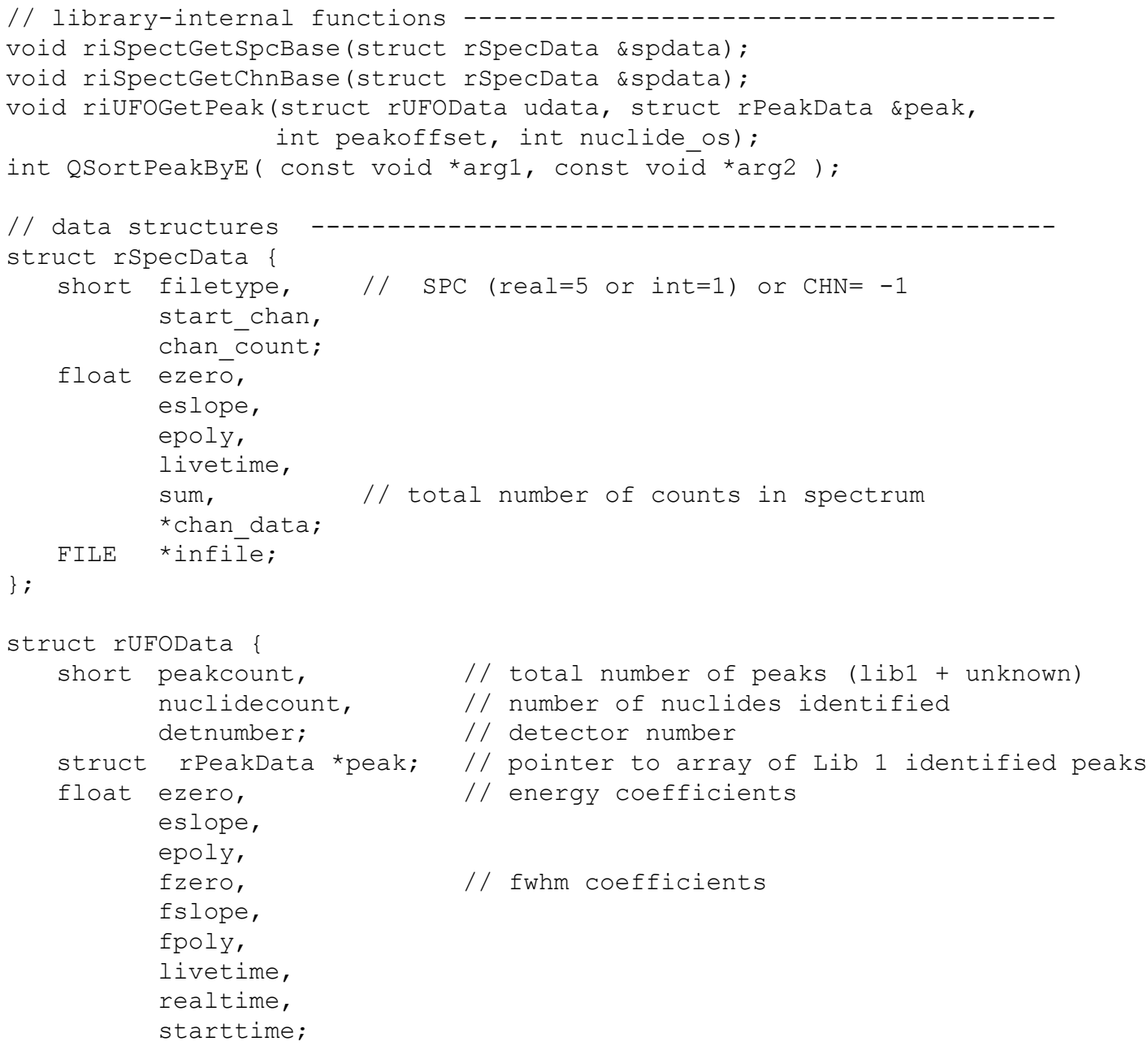

COleDateTime datetime_DATE; 


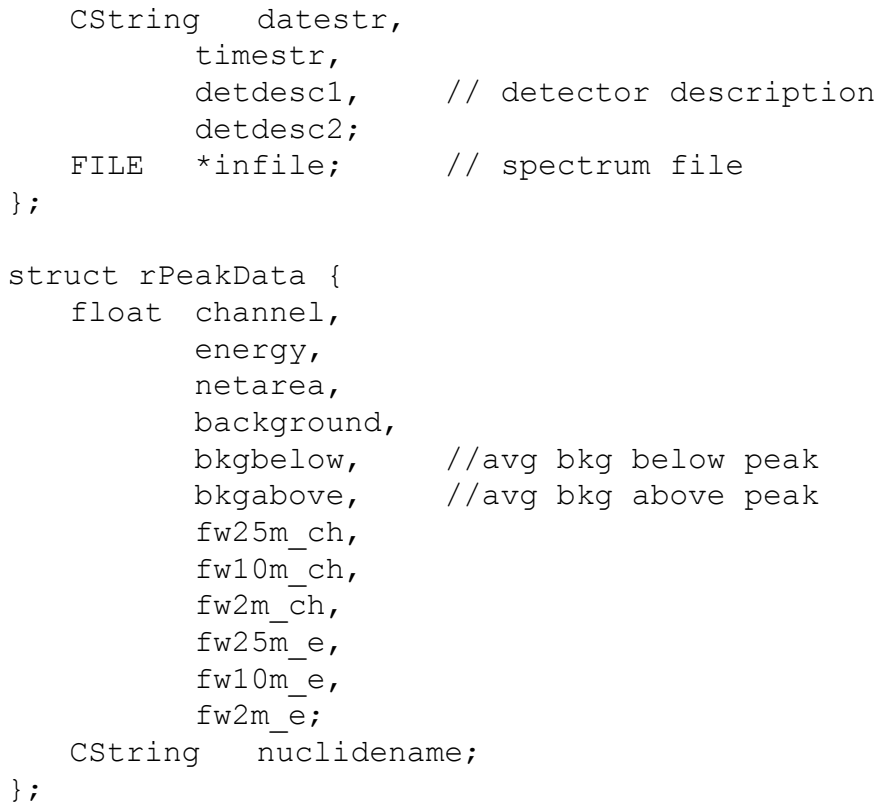


if $(i==\operatorname{SPC} \& \& \quad r t n==1)$

fread (\&spdata.filetype,2,1,spdata.infile); //real=5, int=1 rispectGetSpcBase (spdata);

if $(r \ln ==1)$ spdata. sum=ArraySum (spdata. chan data, 0 , spdata. chan count-1) fclose (spdata.infile):

\}

return rtn;

void riSpectGetChnBase (rSpecData \&spdata)

short chan offset; // bytes from beginning of file to start of chan data int temp;

FILE *infile = spdata.infile;

fseek (infile, 12, SEEK SET);

fread(\&temp,4,1,infile); // chn time stored as number of $20 \mathrm{~ms}$ ticks

spdata.livetime $=($ float $)$ temp*20/1000

fseek (infile, 28, SEEK SET)

fread (\&spdata.start_chan, 2, 1, infile);

fread (\&spdata.chan_count, 2,1,infile);

chan offset $=32$;

int* ichan data $=$ new int [spdata.chan_count];

fseek (infile, chan offset, SEEK SET)

fread (ichan_data, $\overline{4}$, spdata.chañ_count, infile);

spdata.chan_data=ArrayCopy (ichan_data, spdata.chan_count) ;

delete[] ichan data,

fseek (infile, 4, SEEK_CUR);

// get energy cal information

fread (\&spdata.ezero, 4,1, infile);

fread (\&spdata.eslope, 4,1,infile);

fread (\&spdata.epoly, 4,1, infile);

return;

void riSpectGetSpcBase (rSpecData \&spdata)

FILE *infile = spdata.infile 
short calrecoffset, chan_offset;

fseek (infile, 94, SEEK_SET);

fread (\&spdata.livetime, 4,1,infile);

fseek (infile, 60,SEEK_SET);

fread (\&chan_offset,2,1,infile); // offset = record count

fseek (infile, 64, SEEK_SET);

fread (\&spdata. chan count, 2, 1, infile);

fread (\&spdata.start_chan, 2, 1, infile);

if (spdata.filetype $==1$ )

\{ // integer SPC file. 128 bytes per record, 4 bytes per channel

int* ichan_data= new int [spdata.chan_count];

fseek (infile, (chan offset-1)*128, SEEK SET);

fread (ichan_data, 4, spdata.chan_count, infile);

spdata.chan_data=ArrayCopy (ichān_data, spdata.chan_count) ;

delete[] ichan data;

else

\{ // real (float) SPC file. 128 bytes per record, 4 bytes per channel

spdata.chan_data $=$ new float [spdata.chan_count];

fseek (infile, (chan offset-1)*128, SEEK SET);

\}

fread (spdata.chan_data,4,spdata.chan_count,infile);

// get energy cal information

fseek (infile, 34,SEEK_SET);

fread (\&calrecoffset, $\overline{2}, 1$, infile);

// note: calrecoffset is the start of the calibration DATA record

fseek (infile, (calrecoffset-1)*128+20,SEEK_SET);

fread (\&spdata.ezero,4,1,infile);

fread (\&spdata.eslope, 4, 1, infile);

fread (\&spdata.epoly, 4,1, infile);

return;

\}

float SpcSumRegione(struct rSpecData spec, float e1, float e2)

\{

// sums counts between e1 $\mathrm{keV}$ and e2 $\mathrm{keV}$ in spectrum

int $\mathrm{cl}=\mathrm{EtoCh}(\mathrm{el}$, spec.ezero, spec.eslope, spec.epoly);

int $c 2=E t o c h(e 2$, spec.ezero, spec.eslope, spec.epoly);

if (c2>(spec.chan_count-1)) c2=spec. chan_count-1; 
// get channel numbers for e1 and e2 using quadradric solution

float ChtoE(float i, float zero, float slope, float poly)

// converts channel count i to energy (keV) given

// calibration coefficients a1=zero, a2=slope and a3=quadradic coeff.

float $a=z e r o, b=s l o p e, c=p o l y, r t n$;

$r \operatorname{tn}=\mathrm{a}+i * \mathrm{~b}+i * i * \mathrm{C}$

if $(r t n<0) \quad r t n=0$

return rtn;

int Etoch(float e, float a1, float a2, float a3)

// converts energy e to channel number

// uses quadradic solution for $a x^{\wedge} 2+b x+c=0$ and always uses a (+) for sqrt term

// calibration coefficients a1=zero, a2=slope and a3=quadradic coeff.

float $a=a 3, b=a 2, c=a 1-e$;

return (int) $((-b+\operatorname{sqrt}(b * b-4 * a * c)) /(2 * a))$;

int UFOGet (char* infilename, rUFOData \&udata)

// return = 1 for success, 0 for incorrect file type,

// -1 for file not found

int $r \mathrm{tn}=1, i, j i$

short peakcount1, peakcountu,

short filetype1, filetype2,

FILE *infile;

if ((infile=fopen (infilename,"rb")) != NULL

/ / check to insure proper file types

fread (\&filetype1,2,1,infile);

fread (\&filetype2,2,1,infile);

if (filetype1 $!=1$ || filetype2 $!=1024$ ) return 0 ;

else

return -1 ; 
udata.infile=infile;
short gen os, //GEN record
peakl os, //lib 1 peak start record offset
unpeak_os, //unknown peak record offset
nuclidē1_os, //lib 1 nuclide start record offset
calrecl os, //cal data record offset
detdesc_os; //det description record offset

// populate base ufo data structure. UFO files use 64 word (128 byte) records fseek (infile, 12, SEEK_SET);

fread (\&detdesc_os, $2, \overline{1}$, infile);

fseek(infile, 34,SEEK_SET);

fread (\&calrec1_os, $2, \overline{1}$, infile);

fseek (infile, 128, SEEK SET):

fread (\&gen_os, 2,1,infīle);

// libl peaks header

fseek (infile, 128+6, SEEK_SET)

fread (\&peak1_os, 2,1,infile);

fread (\&peakcount1,2,1,infile);

peakcount 1 = peakcount $1 / 2$;

// no idea why this is true...

// unknown peaks header

fseek (infile, 128+24, SEEK SET);

fread (\&unpeak_os, 2,1,infīle);

fread (\&peakcountU, 2, 1, infile);

peakcountU $=$ peakcountU $/ 2$;

// no idea why this is true...

// nuclide header

fseek (infile, 128+30,SEEK_SET);

fread (\&nuclidel os, 2,1, infile);

fread (\&udata.nuclidecount, 2, 1, infile)

// detector desc

fseek (infile, (detdesc os-1)*128, SEEK SET);

fread (udata.detdesc1. GetBuffer (64), 64, 1 , infile);

fread (udata.detdesc2. GetBuffer (64), 64,1, infile);

udata.detdesc1.TrimRight ();

udata.detdesc2. TrimRight (),

udata.detdesc1. ReleaseBuffer();

udata.detdesc2. ReleaseBuffer();

// cal data

A-14 


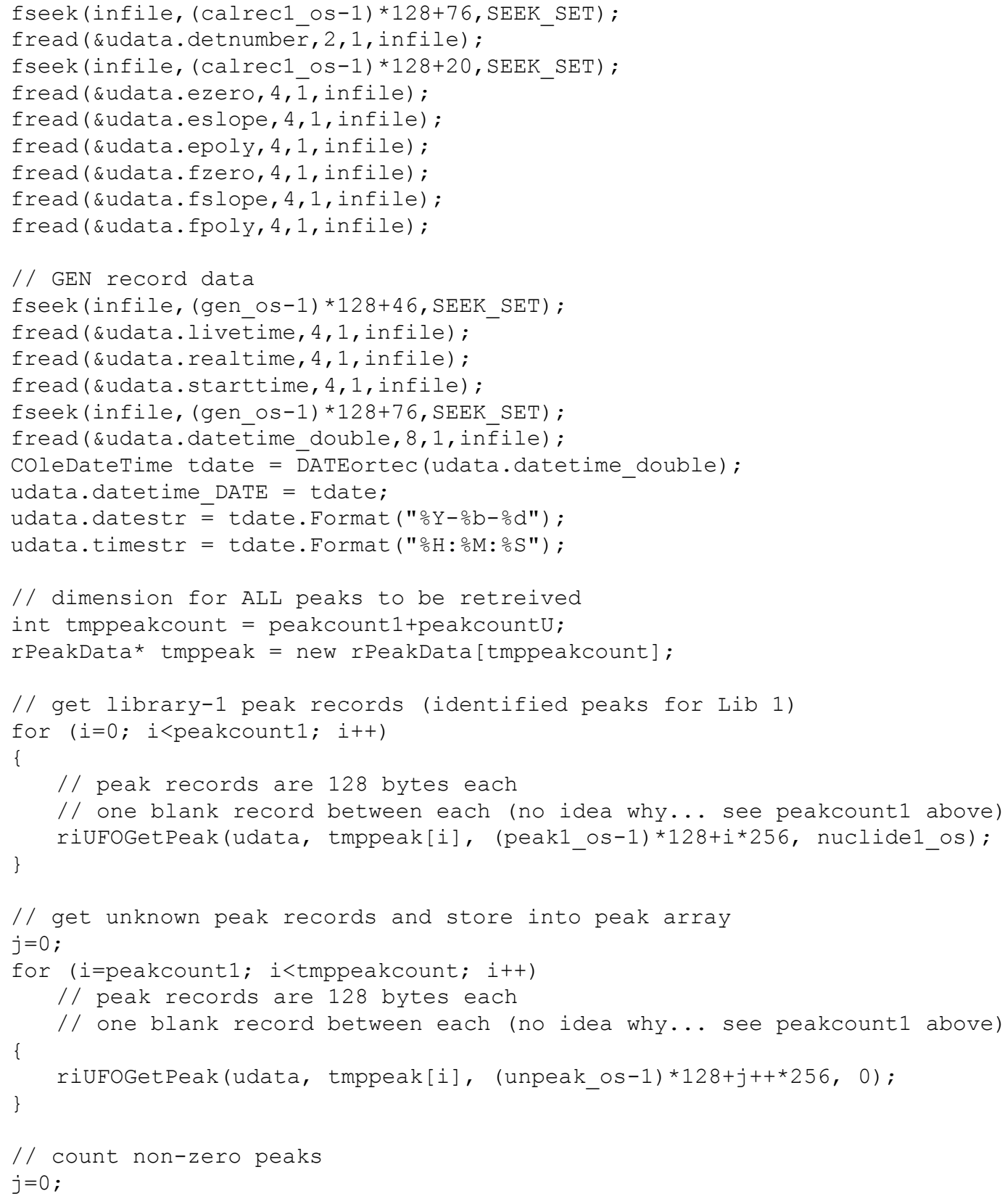




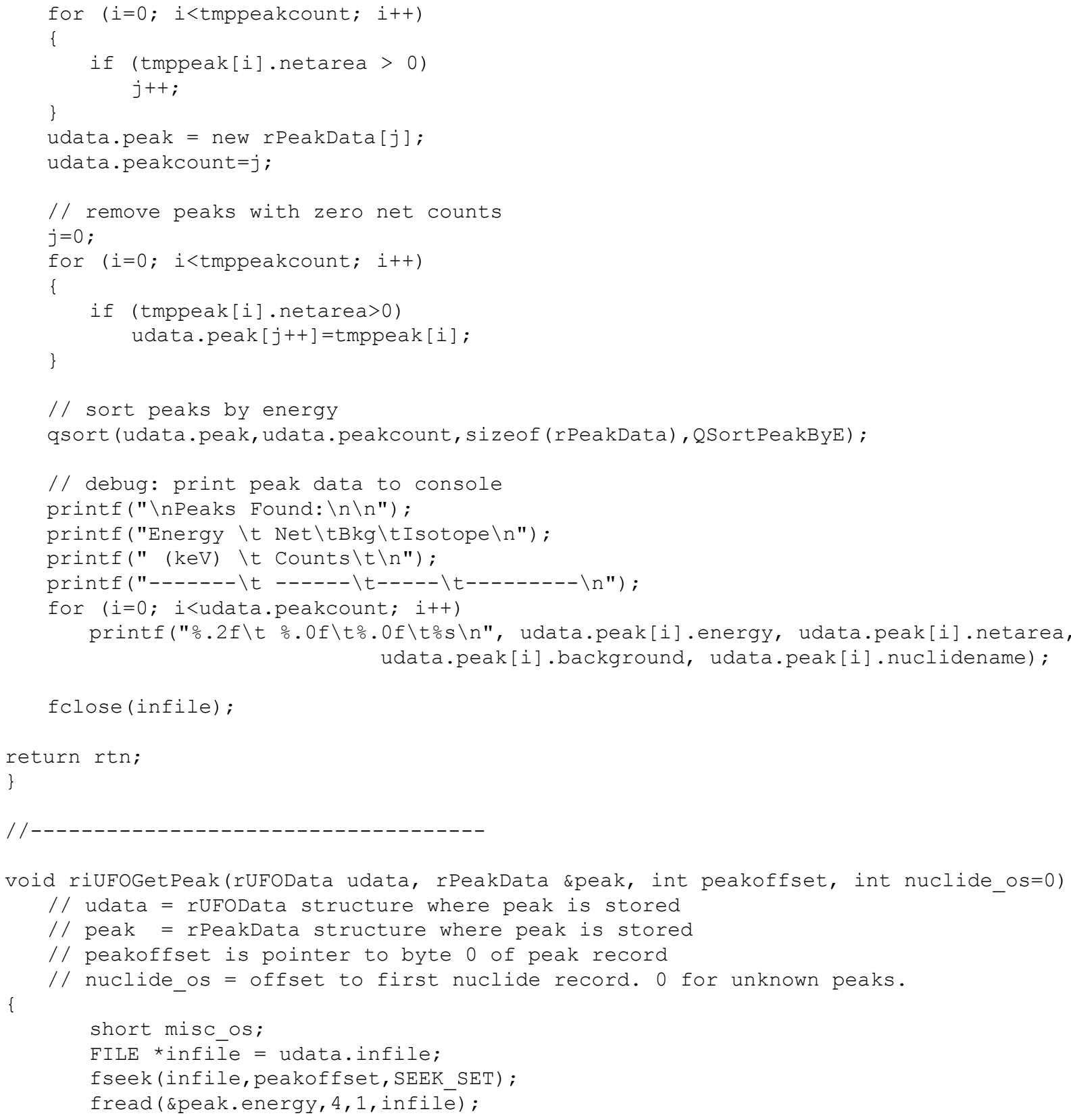

fclose(infile);

// - - - - - - - - - - - - - - - - - - - - - - - - - - - -

void riUfOGetPeak (rUFOData udata, rPeakData \&peak, int peakoffset, int nuclide_os=0)

// udata = rUFOData structure where peak is stored

$/ /$ peak = rPeakData structure where peak is stored

$/ /$ peakoffset is pointer to byte 0 of peak record

// nuclide os = offset to first nuclide record. 0 for unknown peaks.

short misc os;

FILE *infile = udata.infile;

fseek (infile, peakoffset, SEEK_SET) ;

fread (\&peak.energy, 4,1,infilē);

return rtn

\{ 
fseek (infile, peakoffset+12, SEEK SET)

fread (\&peak.netarea, 4, 1, infile);

fread ( \&peak.background, 4, 1, infile);

fseek (infile, peakoffset+28, SEEK SET)

fread (\&peak. channel, 4,1,infile);

fseek (infile, peakoffset+80,SEEK_SET) ;

fread ( \&peak.bkgbelow, 4, 1, infile);

fread ( \&peak.bkgabove, 4, 1, infile)

fseek (infile, peakoffset+32, SEEK_SET) ;

fread (\&peak.fw25m_ch,4,1,infile);

fread (\&peak.fw10m ch, 4, 1, infile)

fread (\&peak.fw2m_ch, 4, 1, infile);

if (peak.netarea <0) peak.netarea $=0$;

if (peak.fw25m_ch $<1$ ) peak.fw25m_ch=0;

if (peak.fw10m ch $<1$ ) peak.fw10m ${ }^{-} h=0$

if (peak.fw2m_ch $<1$ ) peak.fw2m_ch=0;

if (nuclide_os $>0$ )

fseek (infile, peakoffset +48 , SEEK SET)

fread (\&misc_os, 2,1,infile);

/ nuclide records are 64 bytes. nuclide record offset is

// given relative to 128 -byte file records.

fseek (infile, (nuclide_os-1)*128+(misc_os-1)*64,SEEK_SET);

fgets (peak.nuclidename.GetBuffer (8), 8, infile);

peak.nuclidename.ReleaseBuffer();

else

peak.nuclidename="Unknown";

// calculate peak parameters in keV

float a1=udata.ezero, a2=udata.eslope, a3=udata.epoly;

peak.fw25m_e= ChtoE (peak.fw25m_ch, a1, a2, a3);

peak.fw10m e= ChtoE (peak.fw10m ch, a1, a2, a3);

peak.fw2m e = ChtoE (peak.fw2m ch, a1, a2, a3)

int UFOFindPeak (rUFOData \&udata, float e1, float e2)

$1 /$ find peak closest to energy e1 from udata. Must be within +/- e2 of e1.

$/ /$ e1 = target and e2 = tolerance are expressed in $\mathrm{keV}$

// returns index into peak array for udata 
// returns -1 if peak not found

// Example: rUFOData test;

// int $i=$ UFOFindPeak(test, 662, 2);

// //find peak within $2 \mathrm{keV}$ of $662 \mathrm{keV}$

// cout $<$ test.peak[i].netarea

int $i$, select $=-1$

double d1, d2;

for $(i=0 ; i<u d a t a$. peakcount; $i++)$

// raw compare

if ((udata.peak[i].energy $<(e 1+e 2))$ \&\& (udata.peak[i].energy $>(e 1-e 2))$

if ( select $>-1$ )

// compare to previous find to select best pick

\{

$\mathrm{d} 1$ = fabs (udata.peak[select].energy-e1);

$\mathrm{d} 2$ = fabs (udata.peak[i].energy-e1)

if $(\mathrm{d} 2<\mathrm{d} 1)$

\}

select $=i$

lse

$1 /$ first to be found

select $=i$;

return select

\#endif

A-18 


\section{ELECTRONIC DISTRIBUTION}

\section{Internal}

R. L. Coleman

V. M. Baylor

J. S. Bogard

M. E. Murray

ORNL Laboratory Records-CPPR

\section{External}

David P. Spears, U.S. Department of Energy, Office of Nonproliferation Research and Engineering, Routing NA-22, Forrestal GH-068, 1000 Independence Avenue, S.W., Washington, DC 20585

Daniel L. Upp, Vice President, ORTEC, 801 S. Illinois Ave., Oak Ridge, TN 37831 